TRANSACTIONS OF THE

AMERICAN MATHEMATICAL SOCIETY

Volume 352, Number 8, Pages 3523-3555

S 0002-9947(00)02537-X

Article electronically published on March 2, 2000

\title{
ON COHOMOLOGY ALGEBRAS OF COMPLEX SUBSPACE ARRANGEMENTS
}

\author{
EVA MARIA FEICHTNER AND GÜNTER M. ZIEGLER
}

\begin{abstract}
The integer cohomology algebra of the complement of a complex subspace arrangement with geometric intersection lattice is completely determined by the combinatorial data of the arrangement. We give a combinatorial presentation of the cohomology algebra in the spirit of the Orlik-Solomon result on the cohomology algebras of complex hyperplane arrangements. Our methods are elementary: we work with simplicial models for the complements that are induced by combinatorial stratifications of complex space. We describe simplicial cochains that generate the cohomology. Among them we distinguish a linear basis, study cup product multiplication, and derive an algebra presentation in terms of generators and relations.
\end{abstract}

\section{IntRoduction}

Subspace arrangements have attracted interest from topological, algebraic, as well as from combinatorial points of view. It is the interplay of methods from seemingly distant areas that makes the theory of subspace arrangements a vivid and appealing field of research (see the recent surveys by Björner $\mathrm{Bj} 3 \mathrm{Bj} 4$ ). In the present paper we are concerned with the interaction of topological and combinatorial structure of complex subspace arrangements.

Let $\mathcal{A}=\left\{U_{1}, \ldots, U_{n}\right\}$ be a complex subspace arrangement, that is, a finite set of complex linear subspaces in $\mathbb{C}^{d}$. Two topological spaces are naturally associated to the arrangement, the singularity link $\mathcal{V}_{\mathcal{A}}:=\bigcup \mathcal{A} \cap S^{2 d-1}$, and the complement $\mathcal{M}(\mathcal{A}):=\mathbb{C}^{d} \backslash \bigcup \mathcal{A}$. Their homotopy types, homology groups, cohomology algebras, etc. are among the topological invariants of the arrangement. On the other hand, there are combinatorial data associated to the arrangement - the intersection lattice $\mathcal{L}(\mathcal{A})$, defined as the poset of intersections among subspaces in $\mathcal{A}$ ordered by reverse inclusion, and the codimension function $\operatorname{codim}_{\mathbb{C}}: \mathcal{L}(\mathcal{A}) \rightarrow \mathbb{N}$, which assigns to every element in $\mathcal{L}(\mathcal{A})$ the complex codimension of the respective subspace of $\mathbb{C}^{d}$.

It is of interest to see the extent in which the topological invariants of an arrangement are determined by the combinatorial data. Specifically, we ask here:

Received by the editors July 8, 1998.

2000 Mathematics Subject Classification. Primary 52C35, 55N45; Secondary 05B35, 51D25, $57 \mathrm{~N} 80$

The first author was supported by the Graduate School "Algorithmic Discrete Mathematics" in Berlin, DFG grant GRK 219/2-97.

The second author was supported by the DFG Gerhard Hess Prize Zi 475/1-1/2 and by the German-Israeli Foundation grant I-0309-146.06/93. 
- Is the cohomology algebra of the complement of a (complex) subspace arrangement determined by the intersection lattice together with the codimension function?

- Can one give an explicit algebra presentation in terms of generators and relations?

- Can the generators be represented by explicit combinatorial/geometric cocycles, and can the relations be verified in terms of these?

A positive answer to this last question should reveal an explicit correspondence between the topological and the combinatorial structure of an arrangement.

All three questions have satisfactory, positive answers for complex hyperplane arrangements: In a classical paper from 1980, Orlik and Solomon [OS] showed that the cohomology algebra of the complement is completely determined by the combinatorial data of an arrangement. They gave a presentation of the cohomology algebra in generators and relations that depends only on combinatorial data. (In the hyperplane case, the $\operatorname{codim}_{\mathbb{C}}$-function is determined by $\mathcal{L}$.) For their proof they used Brieskorn's $\mathrm{Bn}$ description of the cohomology algebra in terms of explicit de Rham classes, relying on Lefschetz type arguments from algebraic geometry. Later, an elementary reproof by Björner and Ziegler [BZ] complemented the OrlikSolomon result, and provided a positive answer to the third question. Our present investigations are based on [BZ], and we will thus comment on this work in some detail.

The results on cohomology algebras of complements of complex subspace arrangements are less complete: The linear structure was clarified by Goresky and MacPherson [GM, Part III]. Recently, De Concini and Procesi [DP] showed that the rational cohomology algebras of complex subspace arrangements are completely determined by the combinatorial data of the arrangements. They provide rational models for the complements, i.e., differential graded algebras whose cohomology algebras are isomorphic to the rational cohomology algebras of the arrangements. Regarding a description of the algebras in combinatorial terms, their result is far from explicit. The De Concini-Procesi model has been considerably simplified by Yuzvinsky [Y], who conjectures a natural integral version of his model to be an integral model for the complements of complex subspace arrangements $[\mathbf{Y}$, Conj. 6.6].

In the present paper we concentrate on complex subspace arrangements with geometric intersection lattices (in the sense of matroid theory $[\mathrm{CR}] \mathrm{Ox}]$ ); we call them geometric arrangements for brevity. We will describe the integer cohomology algebras of their complements in the spirit of the Orlik-Solomon result for hyperplane arrangements, striving for an elementary and geometrically elucidating proof. We provide an outline of our work in the following.

Our investigations are based on Björner and Ziegler [BZ] in the hyperplane case. We adjust their approach to the study of subspace arrangements: When carefully choosing an appropriate hyperplane arrangement, the induced combinatorial stratification of complex space [BZ, Sect.2] yields cellular models for the link and the complement of a given subspace arrangement. These models are regular CW complexes whose face posets have simple combinatorial descriptions. We study the complement of an arrangement through the barycentric subdivision of its cellular model, thus working within simplicial cohomology theory (Section 2). In the case of arrangements with boolean intersection lattices, boolean arrangements for brevity, we particularly refine our models by a specific choice of inducing hyperplane arrangements (Section 3 ). 
We give explicit descriptions of certain simplicial cocycles on the complements of boolean arrangements - a definition that plays a key role also beyond the boolean case (Section 4). Restriction from the complements of boolean subarrangements transfers these cocycles to complements of general geometric arrangements. We distinguish a linear basis of the cohomology algebra among the resulting cohomology classes (Section 5 ).

Several choices are involved in the definition of our cocycles. We spend most of Section 6 to show that when varying these choices the corresponding cohomology classes are affected at most by a sign change, which we can control. Thus, for distinguished cohomology classes we have a variety of explicit representing cocycles at hand. This allows us to study cup product multiplication and linear relations among the proposed generators of the cohomology algebra. Combined with the linear basis obtained in Section 5, this results in a combinatorial presentation of the integer cohomology algebra in terms of generators and relations (Section 7 . Theorem 7.3).

We close our investigations with some remarks on the specialization of our result to complex hyperplane arrangements and on an extension to real $(\bmod 2)$ arrangements - arrangements of real linear subspaces for which all intersections have even codimensions (Theorem 7.5).

\section{ACKNOWLEDGMENTS}

Sergey Yuzvinsky $[Y]$ independently obtained a combinatorial presentation for the cohomology algebras of geometric subspace arrangements, using his integral models for the complements of complex subspace arrangements. We thank Dmitry

Kozlov for carefully reading an early version of our paper and for detecting a crucial error which led us to the "right" combinatorial stratifications in the boolean case.

\section{Combinatorial Stratifications}

Combinatorial stratifications for complex hyperplane arrangements were introduced in [BZ]. They yield a complete encoding of the arrangement's topology into combinatorial data. Here we review the basic construction and then adjust it to the study of complex subspace arrangements.

Define a sign function $s: \mathbb{C} \longrightarrow\{0,+,-, i, j\}$ on the complex plane:

$$
s(x+i y)=\left\{\begin{aligned}
i & \text { if } y>0, \\
j & \text { if } y<0 \\
+ & \text { if } y=0 \text { and } x>0 \\
- & \text { if } y=0 \text { and } x<0 \\
0 & \text { if } y=x=0 .
\end{aligned}\right.
$$

Let $\mathcal{H}=\left\{H_{1}, \ldots, H_{n}\right\}$ be an arrangement of complex linear hyperplanes in $\mathbb{C}^{d}$. Assume that $\mathcal{H}$ is essential, that is, $\bigcap \mathcal{H}=\{0\}$, and that the hyperplanes are given by complex linear forms $\ell_{i}: \mathbb{C}^{d} \rightarrow \mathbb{C}$ with $H_{i}=\operatorname{ker} \ell_{i}$ for $i=1, \ldots, n$. To each point in $\mathbb{C}^{d}$ we assign a complex sign vector that encodes its relative position to the complex hyperplanes of the arrangement:

$$
\begin{aligned}
s_{\mathcal{H}}: \mathbb{C}^{d} & \longrightarrow\{0,+,-, i, j\}^{n} \\
z & \longmapsto\left(s\left(\ell_{1}(z)\right), \ldots, s\left(\ell_{n}(z)\right)\right) .
\end{aligned}
$$


The non-empty sets $s_{\mathcal{H}}{ }^{-1}(X)$, formed by all the points in $\mathbb{C}^{d}$ that $s_{\mathcal{H}}$ maps to the same sign vector $X$, for $X \in\{0,+,-, i, j\}^{n}$, are relative-open convex polyhedral cones. The resulting partition of $\mathbb{C}^{d}$ is called the combinatorial stratification of $\mathbb{C}^{d}$ induced by the arrangement $\mathcal{H}$. Restriction of this stratification to the unit sphere $S^{2 d-1} \subseteq \mathbb{C}^{d}$ yields a regular $\mathrm{CW}$ decomposition $\Gamma_{\mathcal{H}}$ of $S^{2 d-1}$. Its face poset $P_{\mathcal{H}}$ (extended by a minimal element) is given by

$$
P_{\mathcal{H}}=\left(s_{\mathcal{H}}\left(\mathbb{C}^{d}\right), \leq\right),
$$

where " $\leq$ " denotes the componentwise order of the sign vectors in $s_{\mathcal{H}}\left(\mathbb{C}^{d}\right) \subseteq$ $\{0,+,-, i, j\}^{n}$ that is induced by the following partial order of signs:

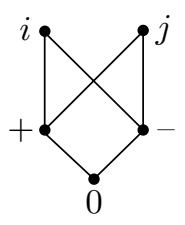

This poset is the (augmented) face poset of the regular CW decomposition of $S^{1}$ induced by $\{0\}$ when considered as a "hyperplane" in $\mathbb{C}^{1}$ :
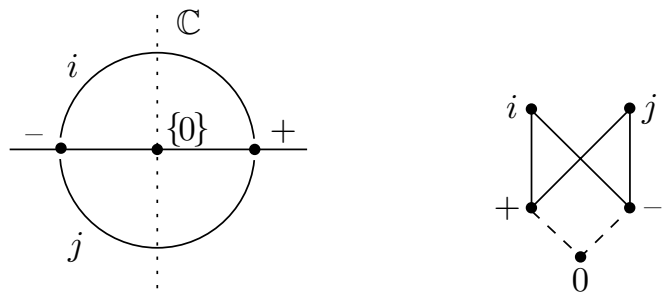

The hyperplanes in $\mathcal{H}$ as well as all of their intersections are unions of strata of the combinatorial stratification. Thus the singularity link $\mathcal{V}_{\mathcal{H}}:=(\bigcup \mathcal{H}) \cap S^{2 d-1}$ is a subcomplex $\Gamma_{\operatorname{link}(\mathcal{H})}$ of $\Gamma_{\mathcal{H}}$, and its augmented face poset $P_{\operatorname{link}(\mathcal{H})}$ is an order ideal of $P_{\mathcal{H}}$. The complement of $P_{\operatorname{link}(\mathcal{H})}$ in $P_{\mathcal{H}}$ is the reversed face poset of a regular CW complex that is a strong deformation retract of (and thus homotopy equivalent to) the complement $\mathcal{M}(\mathcal{H}):=\mathbb{C}^{d} \backslash \cup \mathcal{H}$.

Thus the combinatorial stratification of $\mathbb{C}^{d}$ induced by a hyperplane arrangement leads to cellular models for both the link and the complement of the arrangement. Now we adjust the approach to the study of subspace arrangements.

Let $\mathcal{A}=\left\{U_{1}, \ldots, U_{n}\right\}$ be an arrangement of complex linear subspaces in $\mathbb{C}^{d}$. Call an essential arrangement of complex linear hyperplanes $\mathcal{H}=\left\{H_{i, j}: 1 \leq i \leq n\right.$, $\left.1 \leq j \leq t_{i}\right\}$ in $\mathbb{C}^{d}$ a complex frame of hyperplanes for $\mathcal{A}$ if $\bigcap_{j=1}^{t_{i}} H_{i, j}=U_{i}$ for $i=1, \ldots, n$. Any hyperplane arrangement which contains a complex frame of hyperplanes for $\mathcal{A}$ induces a combinatorial stratification of $\mathbb{C}^{d}$ that leads to cellular models for the link and the complement of the subspace arrangement $\mathcal{A}$.

We will assume that the subspaces of an arrangement $\mathcal{A}=\left\{U_{1}, \ldots, U_{n}\right\}$ in $\mathbb{C}^{d}$ are linearly ordered by the natural order on their index set $[n]:=\{1, \ldots, n\}$, unless stated otherwise. We often consider subsets $\left\{i_{1}, \ldots, i_{k}\right\}$ of the index set; we then use the notation $\left\{i_{1}, \ldots, i_{k}\right\}<$ to indicate that $i_{1}<\ldots<i_{k}$ according to the natural order induced from $[n]$. Moreover, we assume that the hyperplanes in a complex frame $\mathcal{H}=\left\{H_{i, j}: 1 \leq i \leq n, 1 \leq j \leq t_{i}\right\}$ for $\mathcal{A}$ are ordered lexicographically 
with respect to their index pairs $(i, j)$ and that hyperplanes in any subarrangement of a complex frame are endowed with the order inherited from the order of the frame. We denote the subarrangement $\left\{H_{i, 1}, \ldots, H_{i, t_{i}}\right\}$ of $\mathcal{H}$ that corresponds to the subspace $U_{i}$ with $\mathcal{H}_{i}$ for $i=1, \ldots, n$. The hyperplanes in a complex frame need not necessarily be distinct.

To describe the position of a cell $\theta$ in $\Gamma_{\mathcal{H}}$ with respect to a subarrangement, we use the following: By $\theta(H)$ we denote the sign vector entry for $\theta$ with respect to the hyperplane $H$ in $\mathcal{H}$. For a sequence of hyperplanes $\mathcal{H}_{0}=\left(H_{1}, \ldots, H_{k}\right)$ in $\mathcal{H}$ we abbreviate the sequence of sign vector entries $\left(\theta\left(H_{1}\right), \ldots, \theta\left(H_{k}\right)\right)$ with $\theta\left(H_{1}, \ldots, H_{k}\right)$, or even shorter with $\theta\left(\mathcal{H}_{0}\right)$.

Our setting in the following will consist of a complex subspace arrangement $\mathcal{A}=\left\{U_{1}, \ldots, U_{n}\right\}$ such that the subspaces in $\mathcal{A}$ are pairwise distinct and inclusion maximal, together with a hyperplane arrangement $\mathcal{G}$ that contains a complex frame $\mathcal{H}=\mathcal{H}_{1} \cup \ldots \cup \mathcal{H}_{n}$ for $\mathcal{A}$. If we were concerned about economical cell decompositions, then we should require that $\mathcal{G}=\mathcal{H}$ is a minimal complex frame, that is, a complex frame with $\left|\mathcal{H}_{i}\right|=\operatorname{codim}_{\mathbb{C}} U_{i}$ for $i=1, \ldots, n$. However, the present set-up simplifies inductive proofs.

The condition on $\mathcal{G}$ ensures that the cells in $\Gamma_{\mathcal{G}}$ that are contained in the link of $\mathcal{A}$ form a subcomplex $\Gamma_{\operatorname{link}(\mathcal{A})}$ of $\Gamma_{\mathcal{G}}$ - a combinatorial cell decomposition for the link of the subspace arrangement. The cells in $\Gamma_{\operatorname{link}(\mathcal{A})}$ are easily characterized in terms of their sign vectors (we switch freely between cells of the decomposition and their encoding in sign vectors):

$$
\theta \in \Gamma_{\operatorname{link}(\mathcal{A})} \Longleftrightarrow \theta\left(\mathcal{H}_{i}\right)=(0, \ldots, 0) \quad \text { for some } i \in\{1, \ldots, n\} .
$$

We denote the (augmented) face poset of $\Gamma_{\operatorname{link}(\mathcal{A})}$ by $P_{\operatorname{link}(\mathcal{A})}$.

The strata in $\mathbb{C}^{d}$ defined by $\mathcal{G}$ are relative-open polyhedral cones, which implies that $\Gamma_{\mathcal{G}}$ is a PL decomposition of the unit sphere [BZ, Thm. 2.6]. Thus, the following applies to $\Gamma_{\mathcal{G}}$ :

Lemma 2.1 ( $\overline{\mathrm{BZ}}$ Prop. 3.1]). Let $P$ be the face poset of a PL regular $C W$ decomposition $\Gamma$ of a sphere. Let $P_{0}$ be a lower ideal in $P$, the face poset of a subcomplex $\Gamma_{0}$ in $\Gamma$. Then $\left(P \backslash P_{0}\right)^{\mathrm{op}}$, the poset $P \backslash P_{0}$ under reversed order, is the face poset of a regular $C W$ complex that is a strong deformation retract of $|\Gamma| \backslash\left|\Gamma_{0}\right|$.

We conclude that

$$
P_{\operatorname{cpl}(\mathcal{A})}:=\left(P_{\mathcal{G}} \backslash P_{\operatorname{link}(\mathcal{A})}\right)^{\mathrm{op}}
$$

is the face poset of a regular $\mathrm{CW}$ complex $\Gamma_{\operatorname{cpl}(\mathcal{A})}$ that is homotopy equivalent to $\left|\Gamma_{\mathcal{G}}\right| \backslash\left|\Gamma_{\operatorname{link}(\mathcal{A})}\right|$ - a cellular model for the complement of the arrangement. In fact, $\Gamma_{\operatorname{cpl}(\mathcal{A})}$ is a subcomplex of the dual block complex (cf. [Mu,$\left.\S 64\right]$ ) of $\Gamma_{\mathcal{G}}$ which, because of $\Gamma_{\mathcal{G}}$ being PL, is itself a cell complex. $\Gamma_{\operatorname{cpl}(\mathcal{A})}$ is formed by cells dual to those cells of $\Gamma_{\mathcal{G}}$ that lie in the complement of $\mathcal{A}$ :

$$
\theta^{\mathrm{op}} \in \Gamma_{\operatorname{cpl}(\mathcal{A})} \Longleftrightarrow \theta\left(\mathcal{H}_{i}\right) \neq(0, \ldots, 0) \quad \text { for all } i=1, \ldots, n \text {. }
$$

From the cellular model for the complement we obtain a simplicial model by barycentric subdivision, that is, by considering the order complex of $P_{\operatorname{cpl}(\mathcal{A})}$ :

$$
\Delta\left(P_{\operatorname{cpl}(\mathcal{A})}\right) \cong \operatorname{sd} \Gamma_{\operatorname{cpl}(\mathcal{A})} \simeq \mathcal{M}(\mathcal{A})
$$

This model is convenient since we can picture simplices in $\Delta\left(P_{\operatorname{cpl}(\mathcal{A})}\right)=$ $\Delta\left(P_{\mathcal{G}} \backslash P_{\operatorname{link}(\mathcal{A})}\right)$ as chains of cells in $\Gamma_{\mathcal{G}} \backslash \Gamma_{\operatorname{link}(\mathcal{A})}$ ordered by inclusion, and thus 
there is no need to switch to dual cells. Furthermore, we prefer a simplicial model for the computation of cup products.

Simplicial cocycles on $\Delta\left(P_{\mathcal{G}} \backslash P_{\operatorname{link}(\mathcal{A})}\right)$ will be constructed as sums of elementary cochains, that is, of cochains that evaluate to 1 on one specified simplex of $\Delta\left(P_{\mathcal{G}} \backslash P_{\operatorname{link}(\mathcal{A})}\right)$ and yield 0 on all others. To specify a simplex in $\Delta\left(P_{\mathcal{G}} \backslash P_{\operatorname{link}(\mathcal{A})}\right)$ means to specify a chain of cells in $\Gamma_{\mathcal{G}} \backslash \Gamma_{\operatorname{link}(\mathcal{A})}$ ordered by inclusion. We introduce notation for this purpose.

Definition 2.2. Let $\left(H_{1}, \ldots, H_{k}\right)$ be a sequence of hyperplanes in $\mathcal{G}$. A chain of cells $\theta: \theta^{(0)}<\ldots<\theta^{(2 k-1)}$ in $\Gamma_{\mathcal{G}}$ is elementary ascending along $\left(H_{1}, \ldots, H_{k}\right)$ if for $1 \leq r \leq k$ and $0 \leq s \leq 2 k-1$

$$
\theta^{(s)}\left(H_{r}\right)=\left\{\begin{array}{cc}
0 & \text { if } s<2 r-2, \\
+ & \text { if } s=2 r-2, \\
i & \text { if } s>2 r-2 .
\end{array}\right.
$$

More explicitly, the following pattern must occur in the sign vectors for the cells in $\theta$ :

$\begin{array}{lcccccc} & H_{1} & H_{2} & H_{3} & H_{4} & \ldots & H_{k} \\ \theta^{(2 k-1)} & i & i & i & i & \ldots & i \\ \theta^{(2 k-2)} & i & i & i & i & \ldots & + \\ \vdots & \vdots & \vdots & & & & \vdots \\ \theta^{(3)} & i & i & 0 & 0 & \ldots & 0 \\ \theta^{(2)} & i & + & 0 & 0 & \ldots & 0 \\ \theta^{(1)} & i & 0 & 0 & 0 & \ldots & 0 \\ \theta^{(0)} & + & 0 & 0 & 0 & \ldots & 0\end{array}$

Elementary ascending chains are the building blocks for the following:

Definition 2.3. Let $\mathcal{F}_{t}=\left(F_{t, 1}, \ldots, F_{t, d_{t}}\right), t=1, \ldots, m$, be sequences of hyperplanes, all contained in the arrangement $\mathcal{G}$. A chain of cells $\theta: \theta^{(0)}<\ldots<$ $\theta^{\left(2 \sum d_{t}-m\right)}$ in $\Gamma_{\mathcal{G}}$ is ascending along $\left(\mathcal{F}_{1}, \ldots, \mathcal{F}_{m}\right)$ if

$$
\theta^{(0)}\left(\mathcal{F}_{t}\right)=(+, 0, \ldots, 0) \quad \text { for } t=1, \ldots, m,
$$

and if for each $t, 1 \leq t \leq m$, the chain

$$
\theta^{(D(t)+1)}<\theta^{(D(t)+2)}<\ldots<\theta^{\left(D(t)+2 d_{t}\right)} \quad \text { for } D(t):=2\left(\sum_{j=1}^{t-1} d_{j}\right)-t
$$

is elementary ascending along $\left(F_{t, 1}, \ldots, F_{t, d_{t}}\right)$. 
We again illustrate this definition with picturing sign vector entries for an ascending chain.

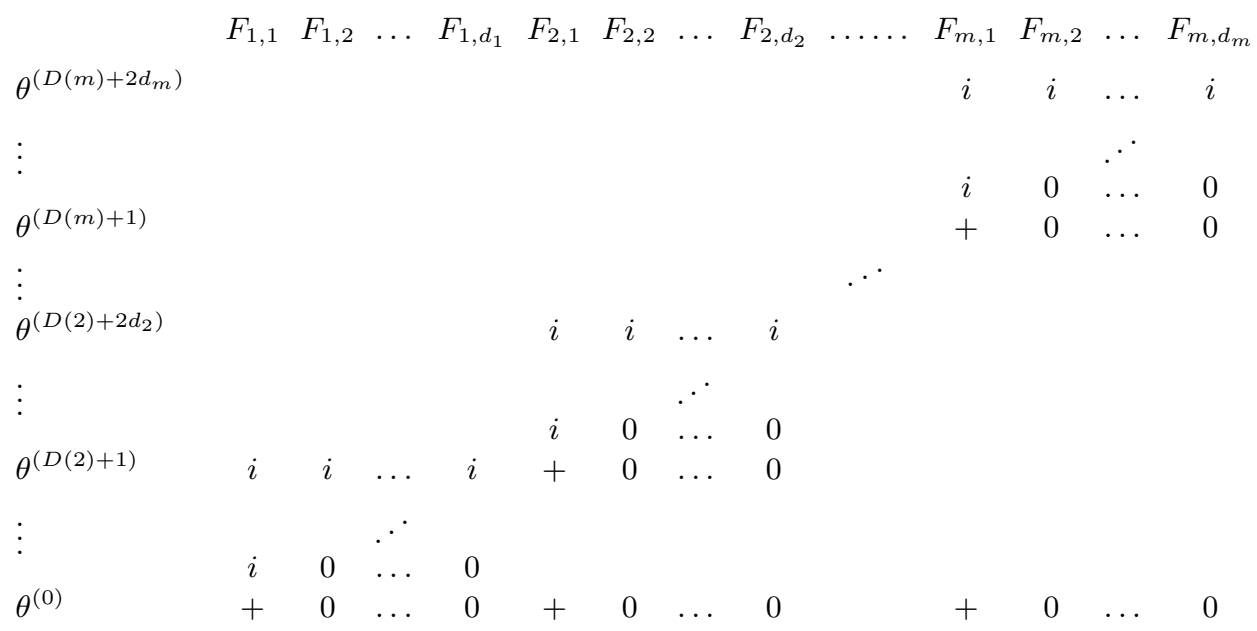

Ascending chains will play a crucial role for the construction of cocycles on $\Delta\left(P_{\mathcal{G}} \backslash P_{\operatorname{link}(\mathcal{A})}\right)$.

\section{StRATifications FOR BOOLEAN ARRANGEMENTS}

The next two sections concern boolean arrangements: We use special complex frames and their induced stratifications for the construction of explicit simplicial cocycles. These cocycles will then be transferred to general geometric subspace arrangements.

An arrangement $\mathcal{A}=\left\{U_{1}, \ldots, U_{n}\right\}$ of complex linear subspaces in $\mathbb{C}^{d}$ is boolean if its intersection lattice is a boolean algebra $\mathcal{B}_{n}$ on $n$ elements, that is, if the $2^{n}$ intersections $U_{S}:=\bigcap_{i \in S} U_{i}$ for $S \subseteq[n]$ are pairwise distinct.

Definition 3.1. Let $\mathcal{A}=\left\{U_{1}, \ldots, U_{n}\right\}$ be a boolean arrangement in $\mathbb{C}^{d}$. Choose vectors $u_{i}$ with $u_{i} \in\left(\bigcap_{j \neq i} U_{j}\right) \backslash U_{i}$ for $i=1, \ldots, n$, and set

$$
V_{i}:=\operatorname{span}_{\mathbb{C}}\left(U_{i} \cup\left\{u_{i}\right\}\right) .
$$

Then $\mathcal{A}_{\triangle}:=\left\{V_{1}, \ldots, V_{n}\right\}$ is a framing arrangement for $\mathcal{A}$.

The choice of the vectors $u_{i}$ is possible if (and only if!) we deal with a boolean arrangement.

Proposition 3.2. Let $\mathcal{A}_{\triangle}=\left\{V_{1}, \ldots, V_{n}\right\}$ be a framing arrangement for the boolean arrangement $\mathcal{A}=\left\{U_{1}, \ldots, U_{n}\right\}$ in $\mathbb{C}^{d}$. Then

$$
\operatorname{dim}_{\mathbb{C}}\left(V_{S}\right)-\operatorname{dim}_{\mathbb{C}}\left(U_{S}\right)=|S| \quad \text { for } S \subseteq[n] .
$$

Proof. The statement is obvious for $|S|=0,1$. For $S=\left\{i_{1}, \ldots, i_{k}\right\}, k>1$, consider the following sequence of inclusions:

$$
\begin{aligned}
U_{i_{1}} \cap \ldots \cap U_{i_{k}} & \subseteq V_{i_{1}} \cap U_{i_{2}} \cap \ldots \cap U_{i_{k-1}} \cap U_{i_{k}} \\
& \vdots \\
& \subseteq V_{i_{1}} \cap V_{i_{2}} \cap \ldots \cap V_{i_{k-1}} \cap U_{i_{k}} \subseteq V_{i_{1}} \cap \ldots \cap V_{i_{k}} .
\end{aligned}
$$

By the very construction of the subspaces $V_{i}$ from the subspaces $U_{i}$, the difference in dimension is exactly 1 for each of the inclusions. 
Definition 3.3. Let $\mathcal{A}=\left\{U_{1}, \ldots, U_{n}\right\}$ be a boolean arrangement in $\mathbb{C}^{d}$ with framing arrangement $\mathcal{A}_{\triangle}=\left\{V_{1}, \ldots, V_{n}\right\}$, and let $\mathcal{H}=\mathcal{H}_{1} \cup \ldots \cup \mathcal{H}_{n}$ with $\mathcal{H}_{i}=$ $\left\{H_{i, 1}, \ldots, H_{i, t_{i}}\right\}, t_{i} \in \mathbb{N}$ for $i=1, \ldots, n$, be a complex frame for $\mathcal{A}_{\triangle}$. Choose hyperplanes $H_{i, 0}$ in $\mathbb{C}^{d}$ with $H_{i, 0} \cap V_{i}=U_{i}$, and set

$$
\widetilde{\mathcal{H}}=\widetilde{\mathcal{H}}_{1} \cup \ldots \cup \widetilde{\mathcal{H}}_{n} \quad \text { with } \quad \widetilde{\mathcal{H}}_{i}:=\left\{H_{i, 0}\right\} \cup \mathcal{H}_{i}
$$

for $i=1, \ldots, n$. Then $\widetilde{\mathcal{H}}$ is a strong complex frame for the boolean arrangement $\mathcal{A}$ with respect to the framing arrangement $\mathcal{A}_{\triangle}$.

In the setting of Definition 3.3 let $S=\left\{i_{1}, \ldots, i_{k}\right\}_{<} \subseteq[n]$ be a non-empty (ordered) subset of the index set of $\mathcal{A}$. We select hyperplanes from $\mathcal{H}_{i_{1}} \cup \ldots \cup \mathcal{H}_{i_{k}}$, a complex frame for the subarrangement $\mathcal{A}_{\triangle, S}=\left\{V_{i}\right\}_{i \in S}$ of $\mathcal{A}_{\triangle}$ : Choose subsets $\mathcal{F}_{i_{j}}^{S} \subseteq \mathcal{H}_{i_{j}}$ for $j=1, \ldots, k$ by considering the hyperplanes in $\mathcal{H}_{i_{1}} \cup \ldots \cup \mathcal{H}_{i_{k}}$ one by one under reversed linear order, and take $H_{i_{j}, t} \in \mathcal{H}_{i_{j}}$ to belong to $\mathcal{F}_{i_{j}}^{S}$ if it does not contain the intersection of all previously chosen hyperplanes:

$$
\mathcal{F}_{i_{j}}^{S}=\left\{H_{i_{j}, t} \in \mathcal{H}_{i_{j}}: H_{i_{j}, t} \nsupseteq\left(\bigcap_{u>t} H_{i_{j}, u}\right) \cap\left(V_{i_{j+1}} \cap \ldots \cap V_{i_{k}}\right)\right\}, \quad j=1, \ldots, k .
$$

Our selection ensures that the $\mathcal{F}_{i_{j}}^{S}, j=1, \ldots, k$, are pairwise disjoint sets of hyperplanes. To stress that they come along with a natural order inherited from the order on the hyperplanes of the strong complex frame, we refer to them as sequences of hyperplanes.

Definition 3.4. Let $\mathcal{A}=\left\{U_{1}, \ldots, U_{n}\right\}$ be a boolean arrangement in $\mathbb{C}^{d}$ with framing arrangement $\mathcal{A}_{\triangle}=\left\{V_{1}, \ldots, V_{n}\right\}$, and $\widetilde{\mathcal{H}}=\widetilde{\mathcal{H}}_{1} \cup \ldots \cup \widetilde{\mathcal{H}}_{n}$ a strong complex frame for $\mathcal{A}$ with respect to $\mathcal{A}_{\triangle}$. For $\emptyset \neq S=\left\{i_{1}, \ldots, i_{k}\right\}<\subseteq[n]$ choose sequences of hyperplanes $\mathcal{F}_{i_{j}}^{S}, j=1, \ldots, k$, in the complex frame $\mathcal{H}$ for $\mathcal{A}_{\triangle}$ as described above, and set

$$
\widetilde{\mathcal{F}}_{i_{j}}^{S}:=\left\{H_{i_{j}, 0}\right\} \cup \mathcal{F}_{i_{j}}^{S} \quad \text { for } j=1, \ldots, k .
$$

We call $\left(\widetilde{\mathcal{F}}_{i_{1}}^{S}, \ldots, \widetilde{\mathcal{F}}_{i_{k}}^{S}\right)$ the characteristic sequences of hyperplanes for the index set $S$ with respect to the strong complex frame $\widetilde{\mathcal{H}}$ for $\mathcal{A}$.

By definition, the following identities hold for the intersections of characteristic sequences of hyperplanes:

$$
\bigcap_{j=r}^{k} \widetilde{\mathcal{F}}_{i_{j}}^{S}=U_{i_{r}} \cap \ldots \cap U_{i_{k}} \quad \text { for } r=1, \ldots, k .
$$

Using Proposition 3.2 , we deduce that

$$
\begin{aligned}
\left|\widetilde{\mathcal{F}}_{i_{j}}^{S}\right| & =\operatorname{codim}_{\mathbb{C}}\left(V_{i_{j}} \cap \ldots \cap V_{i_{k}}\right)-\operatorname{codim}_{\mathbb{C}}\left(V_{i_{j+1}} \cap \ldots \cap V_{i_{k}}\right)+1 \\
& =\operatorname{codim}_{\mathbb{C}}\left(U_{i_{j}} \cap \ldots \cap U_{i_{k}}\right)-\operatorname{codim}_{\mathbb{C}}\left(U_{i_{j+1}} \cap \ldots \cap U_{i_{k}}\right) \text { for } j=1, \ldots, k .
\end{aligned}
$$

In particular, the union of the $\widetilde{\mathcal{F}}_{i_{j}}^{S}$ is a boolean hyperplane arrangement intersecting in $U_{S}=\bigcap_{i \in S} U_{i}$ - an inclusion minimal complex frame of $U_{S}$, contained in $\widetilde{\mathcal{H}}_{i_{1}} \cup \ldots \cup \widetilde{\mathcal{H}}_{i_{k}}$.

For a subspace arrangement $\mathcal{A}=\left\{U_{1}, \ldots, U_{n}\right\}$ in $\mathbb{C}^{d}$ denote the arrangement obtained by deletion of $U_{n}, \mathcal{A} \backslash\left\{U_{n}\right\}=\left\{U_{1}, \ldots, U_{n-1}\right\}$, by $\mathcal{A}^{\prime}$, and the arrangement obtained by restriction to $U_{n}, \mathcal{A}_{\left\lceil U_{n}\right.}=\left\{U_{1} \cap U_{n}, \ldots, U_{n-1} \cap U_{n}\right\} \subseteq \mathbb{C}^{d}$, by $\mathcal{A}^{\prime \prime}$. In the literature, $\mathcal{A}^{\prime \prime}$ is commonly considered as an arrangement in $U_{n}$, but we need 
to consider it as an arrangement in $\mathbb{C}^{d}$. Throughout this paper, both deletions and restrictions will exclusively be performed with respect to the last subspace of an arrangement. We agree to refer to these special operations when talking about the deletion and the restriction of $\mathcal{A}$.

For any boolean arrangement both its deletion and its restriction are boolean arrangements. We need that the concepts introduced in this section are compatible with deletion and restriction on boolean arrangements: Consider a boolean arrangement $\mathcal{A}=\left\{U_{1}, \ldots, U_{n}\right\}$ with framing arrangement $\mathcal{A}_{\triangle}=\left\{V_{1}, \ldots, V_{n}\right\}$, defined by choice of $u_{i}(\mathcal{A}) \in\left(\bigcap_{j \neq i} U_{j}\right) \backslash U_{i}$ for $i=1, \ldots, n$, and a strong complex frame $\widetilde{\mathcal{H}}=\widetilde{\mathcal{H}}_{1} \cup \ldots \cup \widetilde{\mathcal{H}}_{n}$ with respect to $\mathcal{A}_{\triangle}$.

For $\mathcal{A}^{\prime}, \mathcal{A}_{\triangle}^{\prime}=\left\{V_{1}, \ldots, V_{n-1}\right\}$ is a framing arrangement, and $\widetilde{\mathcal{H}}^{\prime}=\widetilde{\mathcal{H}}_{1} \cup \ldots \cup$ $\widetilde{\mathcal{H}}_{n-1}$ a strong complex frame with respect to $\mathcal{A}_{\triangle}^{\prime}$. Framing arrangements and strong complex frames for all subarrangements of $\mathcal{A}$ can be obtained this way.

We choose

$$
u_{i}\left(\mathcal{A}^{\prime \prime}\right):=u_{i}(\mathcal{A}) \in\left(\bigcap_{j \neq i} U_{j}\right) \backslash U_{i} \subseteq\left(\bigcap_{j \neq i, n} U_{j} \cap U_{n}\right) \backslash\left(U_{i} \cap U_{n}\right), \quad i=1, \ldots, n-1,
$$

and we thus obtain a framing arrangement $\mathcal{A}_{\triangle}^{\prime \prime}=\left\{V_{1}^{\prime \prime}, \ldots, V_{n-1}^{\prime \prime}\right\}$ for $\mathcal{A}^{\prime \prime}$ with $V_{i}^{\prime \prime}:=\operatorname{span}_{\mathbb{C}}\left(U_{i} \cap U_{n},\left\{u_{i}\right\}\right)=V_{i} \cap U_{n}$ for $i=1, \ldots, n-1$. We define a complex frame $\mathcal{H}^{\prime \prime}$ for $\mathcal{A}_{\triangle}^{\prime \prime}$ by setting $\mathcal{H}_{i}^{\prime \prime}:=\mathcal{H}_{i} \cup \widetilde{\mathcal{H}}_{n}$ for $i=1, \ldots, n-1$. Extending $\mathcal{H}_{i}^{\prime \prime}$ to $\widetilde{\mathcal{H}}_{i}^{\prime \prime}:=\left\{H_{i, 0}\right\} \cup \mathcal{H}_{i}^{\prime \prime}$ yields a strong complex frame $\widetilde{\mathcal{H}}^{\prime \prime}=\widetilde{\mathcal{H}}_{1}^{\prime \prime} \cup \ldots \cup \widetilde{\mathcal{H}}_{n-1}^{\prime \prime}$ for $\mathcal{A}^{\prime \prime}$ with respect to $\mathcal{A}_{\triangle}^{\prime \prime}$.

Thus if $\mathcal{G}$ contains a strong complex frame $\widetilde{\mathcal{H}}$ for $\mathcal{A}$, then it also contains strong complex frames for the deletion and the restriction of $\mathcal{A}$, namely $\widetilde{\mathcal{H}}^{\prime}$ and $\widetilde{\mathcal{H}}^{\prime \prime}$ as defined above. It simultaneously induces stratifications of $\mathbb{C}^{d}$ that are suitable for the study of $\mathcal{A}, \mathcal{A}^{\prime}$ and $\mathcal{A}^{\prime \prime}$. We close this section with a comparison of characteristic sequences of hyperplanes in the respective strong complex frames. The proof is straightforward and thus omitted.

Proposition 3.5. Let $\mathcal{A}=\left\{U_{1}, \ldots, U_{n}\right\}$ be a boolean arrangement in $\mathbb{C}^{d}, \widetilde{\mathcal{H}}=$ $\widetilde{\mathcal{H}}_{1} \cup \ldots \cup \widetilde{\mathcal{H}}_{n}$ a strong complex frame with respect to a framing arrangement $\mathcal{A}_{\triangle}$. Consider the induced framing arrangements and strong complex frames for the deletion and the restriction of $\mathcal{A}$ as described above. Then the characteristic sequences of hyperplanes in the respective frames with index set $S=\left\{i_{1}, \ldots, i_{k}\right\}_{<} \subseteq[n-1]$ are given by

$$
\begin{aligned}
& \text { (i) } \widetilde{\mathcal{F}}_{i_{j}}^{S}\left(\mathcal{A}^{\prime}\right)=\widetilde{\mathcal{F}}_{i_{j}}^{S}(\mathcal{A}) \quad \text { for } j=1, \ldots, k, \\
& \text { (ï) } \quad \widetilde{\mathcal{F}}_{i_{j}}^{S}\left(\mathcal{A}^{\prime \prime}\right)= \begin{cases}\widetilde{\mathcal{F}}_{i_{k}}^{S \cup\{n\}}(\mathcal{A}) \cup \widetilde{\mathcal{F}}_{n}^{S \cup\{n\}}(\mathcal{A}) & \text { for } j=k, \\
\widetilde{\mathcal{F}}_{i_{j}}^{S \cup\{n\}}(\mathcal{A}) & \text { for } j<k .\end{cases}
\end{aligned}
$$

\section{Cocycles for boolean arrangements}

Now we combine the prerequisites of Sections 2 and 3 to construct certain cochains on the simplicial models for the complements of boolean arrangements.

Definition 4.1. Let $\mathcal{A}=\left\{U_{1}, \ldots, U_{n}\right\}$ be a boolean subspace arrangement in $\mathbb{C}^{d}$, $\widetilde{\mathcal{H}}$ a strong complex frame for $\mathcal{A}$ with respect to a framing arrangement $\mathcal{A}_{\triangle}$, and $\mathcal{G}$ a hyperplane arrangement that contains $\widetilde{\mathcal{H}}$. 
Each non-empty index set $S=\left\{i_{1}, \ldots, i_{k}\right\}_{<} \subseteq[n]$ corresponds to a subspace $U_{S}:=\bigcap_{i \in S} U_{i}$ of $\mathbb{C}^{d}$. We define a simplicial cochain $c^{S}$ of dimension $2 \operatorname{codim}_{\mathbb{C}} U_{S}-$ $|S|$ on the simplicial model $\Delta\left(P_{\mathcal{G}} \backslash P_{\operatorname{link}(\mathcal{A})}\right)$ for the complement of $\mathcal{A}$ :

$$
c^{S}:=\varepsilon_{|S|} \sum_{\tau} \tau^{*}
$$

Here the sum is over all chains of cells $\tau$ in $\Gamma_{\mathcal{G}} \backslash \Gamma_{\operatorname{link}(\mathcal{A})}$ that are ascending along the characteristic sequences of hyperplanes $\left(\widetilde{\mathcal{F}}_{i_{1}}^{S}, \ldots, \widetilde{\mathcal{F}}_{i_{k}}^{S}\right)$ chosen from $\widetilde{\mathcal{H}}$, while $\tau^{*}$ denotes the elementary cochain that corresponds to the chain $\tau$, and the coefficients are given by

$$
\varepsilon_{|S|}=\left\{\begin{array}{rll}
1 & \text { for }|S| \equiv 0,1(\bmod 4) \\
-1 & \text { for } \quad|S| \equiv 2,3(\bmod 4)
\end{array}\right.
$$

Proposition 4.2. For $\emptyset \neq S \subseteq[n]$, the cochain $c^{S}$ is a cocycle on $\Delta\left(P_{\mathcal{G}} \backslash P_{\operatorname{link}(\mathcal{A})}\right)$.

Proof. Let $l_{S}:=2 \operatorname{codim}_{\mathbb{C}} U_{S}-|S|$ denote the dimension of the cochain $c^{S}$. We evaluate $\delta c^{S}$ on $\left(l_{S}+2\right)$-element chains of cells $\theta: \theta^{(0)}<\ldots<\theta^{\left(l_{S}+1\right)}$ in $\Gamma_{\mathcal{G}} \backslash \Gamma_{\operatorname{link}(\mathcal{A})}$. Denote by $\theta_{j}$ the $\left(l_{S}+1\right)$-element chain obtained from $\theta$ by deletion of the $(j+1)$-th cell for $j=0, \ldots, l_{S}+1$. Then

$$
\delta c^{S}(\theta)=c^{S}(\partial \theta)=\sum_{j=0}^{l_{S}+1}(-1)^{j} c^{S}\left(\theta_{j}\right) .
$$

A non-zero contribution occurs among the $c^{S}\left(\theta_{j}\right)$ if and only if $\theta$ is a 1-cell extension of an $\left(l_{S}+1\right)$-element chain $\eta$ in $\Gamma_{\mathcal{G}} \backslash \Gamma_{\operatorname{link}(\mathcal{A})}$ that is ascending along $\left(\widetilde{\mathcal{F}}_{i_{1}}^{S}, \ldots, \widetilde{\mathcal{F}}_{i_{k}}^{S}\right)$.

With respect to the characteristic sequences of hyperplanes for $S$, the chain $\eta$ has the pattern displayed after Definition 2.3. Thus the pattern of $\theta$, which extends that of $\eta$ by an extra sign vector, either has duplicates on $\widetilde{\mathcal{F}}_{i_{1}}^{S} \cup \ldots \cup \widetilde{\mathcal{F}}_{i_{k}}^{S}$, or it extends by a sign vector that has zero entries with respect to one of the characteristic sequences.

In the first case, for two consecutive cells in $\theta$ the sign vector entries coincide with respect to $\widetilde{\mathcal{F}}_{i_{1}}^{S} \cup \ldots \cup \widetilde{\mathcal{F}}_{i_{k}}^{S}$ and hence two consecutive 1-element deletions $\theta_{j}, \theta_{j \pm 1}$ are ascending along $\left(\widetilde{\mathcal{F}}_{i_{1}}^{S}, \ldots, \widetilde{\mathcal{F}}_{i_{k}}^{S}\right)$. The cochain $c^{S}$ evaluates to \pm 1 with opposite signs on these deletions, whereas no other 1-element deletion of $\theta$ gives a non-trivial contribution under $c^{S}$. Hence, $\delta c^{S}$ evaluates to zero on $\theta$.

In the second case $\theta^{(0)}\left(\widetilde{\mathcal{F}}_{i_{j}}^{S}\right)=(0, \ldots, 0)$ for some $j \in\{1, \ldots, k\}$. As an initial cell of the ascending chain $\eta, \theta^{(1)}\left(\mathcal{F}_{i_{1}}^{S} \cup \ldots \cup \mathcal{F}_{i_{k}}^{S}\right)=(0, \ldots, 0)$ and the same holds for $\theta^{(0)}$. Hence

$$
\theta^{(0)} \subseteq H_{i_{j}, 0} \cap\left(\bigcap_{r=j}^{k} \mathcal{F}_{i_{r}}^{S}\right)=H_{i_{j}, 0} \cap\left(V_{i_{j}} \cap \ldots \cap V_{i_{k}}\right) .
$$

By our construction of strong complex frames, $H_{i_{j}, 0} \cap V_{i_{j}}=U_{i_{j}}$. This implies that $\theta^{(0)} \subseteq U_{i_{j}}$, in contradiction to $\theta$ being a chain of cells in $\Gamma_{\mathcal{G}} \backslash \Gamma_{\operatorname{link}(\mathcal{A})}$.

Remark 4.3. For the final argument in the preceding proof the use of a strong complex frame of hyperplanes is essential: If one takes an arbitrary complex frame in the definition of $c^{S}$, then the cochain $c^{S}$ is not necessarily a cocycle!

Remark 4.4. The definition of the cocycle $c^{S}$ is designed to be compatible with "taking subarrangements": Consider the subarrangement $\mathcal{A}_{T}:=\left\{U_{i}\right\}_{i \in T}$ of $\mathcal{A}$, $S \subseteq T \subseteq[n]$; a framing arrangement and a strong complex frame for $\mathcal{A}_{T}$ are obtained 
by taking subarrangements of the corresponding arrangements for $\mathcal{A}$ (compare Section 3). Obviously, characteristic sequences in the respective strong complex frames coincide for index sets $S \subseteq T$. Let $c_{\mathcal{A}_{T}}^{S}, c_{\mathcal{A}}^{S}$ denote the standard cochains defined on the simplicial models $\Delta\left(P_{\mathcal{G}} \backslash P_{\operatorname{link}\left(\mathcal{A}_{T}\right)}\right), \Delta\left(P_{\mathcal{G}} \backslash P_{\operatorname{link}(\mathcal{A})}\right)$ according to Definition 4.1 By their explicit description in terms of elementary cochains we see that $c_{\mathcal{A}_{T}}^{S}$ maps to $c_{\mathcal{A}}^{S}$ when restricted to $\Delta\left(P_{\mathcal{G}} \backslash P_{\operatorname{link}(\mathcal{A})}\right)$ :

$$
i^{\sharp}\left(c_{\mathcal{A}_{T}}^{S}\right)=c_{\mathcal{A}}^{S},
$$

where $i: \Delta\left(P_{\mathcal{G}} \backslash P_{\operatorname{link}(\mathcal{A})}\right) \longrightarrow \Delta\left(P_{\mathcal{G}} \backslash P_{\operatorname{link}\left(\mathcal{A}_{T}\right)}\right)$ denotes the inclusion. In the sequel we will extensively use restrictions of cocycles from the complements of subarrangements to the complement of an initial arrangement.

Our definition of the cocycles $c^{S}$ depends heavily on the simplicial model. More precisely, it depends on the strong complex frame for $\mathcal{A}$, on the hyperplane arrangement $\mathcal{G}$ that induces the stratification, and on the order of the index set $S$ that so far we assumed to be ascending. In the following we will see that these dependencies have very little effect on the cohomology classes which are represented by the cocycles $c^{S}$ (cf. Propositions 4.5, 6.1) and 6.6).

In the next proposition we fix a strong complex frame $\widetilde{\mathcal{H}}$ for $\mathcal{A}$, and we verify that the cohomology class $\left[c^{S}\right]$ represented by the cocycle $c^{S}$ is independent of the choice of a hyperplane arrangement $\mathcal{G}$ if it only contains $\widetilde{\mathcal{H}}$. This is the only point where we deal with different models for the complement of an arrangement at the same time. We therefore recall that the realization of the simplicial model $\left|\Delta\left(P_{\operatorname{cpl}(\mathcal{A})}\right)\right|$, homeomorphic to $\left|\Gamma_{\operatorname{cpl}(\mathcal{A})}\right|$, is a deformation retract of $\left|\Gamma_{\mathcal{G}}\right| \backslash\left|\Gamma_{\operatorname{link}(\mathcal{A})}\right|$, and hence of $\mathcal{M}(\mathcal{A}) \cap S^{2 d-1}$. It is in the latter space that we have to compare cohomology classes which are defined with respect to different stratifications.

Proposition 4.5. Let $\mathcal{G} \subseteq \mathcal{G}^{\prime}$ be arrangements of complex hyperplanes, both containing a strong complex frame $\widetilde{\mathcal{H}}$ for the boolean subspace arrangement $\mathcal{A}$. The regular cell complex $\Gamma_{\mathcal{G}^{\prime}}$ induced by $\mathcal{G}^{\prime}$ is a subdivision of the complex $\Gamma_{\mathcal{G}}$ induced by $\mathcal{G}$. Denote the respective simplicial models for the complement by $\Delta\left(P_{\operatorname{cpl}(\mathcal{A}), \mathcal{G}^{\prime}}\right)$

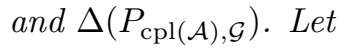

$$
\begin{aligned}
r^{\prime}: \mathcal{M}(\mathcal{A}) \cap S^{2 d-1} & =\left|\Gamma_{\mathcal{G}^{\prime}}\right| \backslash\left|\Gamma_{\operatorname{link}(\mathcal{A}), \mathcal{G}^{\prime}}\right| \longrightarrow\left|\Delta\left(P_{\operatorname{cpl}(\mathcal{A}), \mathcal{G}^{\prime}}\right)\right| \\
r: \mathcal{M}(\mathcal{A}) \cap S^{2 d-1} & =\left|\Gamma_{\mathcal{G}}\right| \backslash\left|\Gamma_{\operatorname{link}(\mathcal{A}), \mathcal{G}}\right|
\end{aligned}
$$

be the deformation retractions from the complement of $\mathcal{A}$ in $S^{2 d-1}$ to the respective realizations of the simplicial models. Then

$$
r^{*}\left(\left[c_{\mathcal{G}^{\prime}}^{S}\right]\right)=r^{*}\left(\left[c_{\mathcal{G}}^{S}\right]\right),
$$

where $c_{\mathcal{G}}^{S}, c_{\mathcal{G}^{\prime}}^{S}$, for $\emptyset \neq S \subseteq[n]$, denote the standard cocycles that are defined in the respective models, both with respect to the strong complex frame $\widetilde{\mathcal{H}}$.

Proof. In any combinatorial stratification of $\mathbb{C}^{d}$ that is appropriate for a study of $\mathcal{A}$, the realization of $\Delta\left(P_{\operatorname{cpl}(\mathcal{A})}\right)$ is the barycentric subdivision of the subcomplex $\Gamma_{\operatorname{cpl}(\mathcal{A})}$ of the dual cell complex of $\Gamma$. It coincides with a subcomplex of the barycentric subdivision of the original cell complex, namely with the realization of $\Delta\left(P \backslash P_{\operatorname{link}(\mathcal{A})}\right)$. For picturing simplices in $\Delta\left(P_{\operatorname{cpl}(\mathcal{A})}\right)$, we will therefore reverse the order on $P_{\operatorname{cpl}(\mathcal{A})}$ and work as before with chains of cells in $\Gamma \backslash \Gamma_{\operatorname{link}(\mathcal{A})}$ ordered by inclusion. The realization of such a simplex in the barycentric subdivision of $\Gamma$ 
is identified with the simplex that is described by the chain of dual cells in the barycentric subdivision of $\Gamma_{\mathrm{cpl}(\mathcal{A})}$.

There is a poset map $f: P_{\mathcal{G}^{\prime}} \backslash P_{\operatorname{link}(\mathcal{A})} \longrightarrow P_{\mathcal{G}} \backslash P_{\operatorname{link}(\mathcal{A})}$ defined on the sign vectors of cells in $\Gamma_{\mathcal{G}^{\prime}} \backslash \Gamma_{\operatorname{link}(\mathcal{A})}$ by "forgetting" the sign entries with respect to hyperplanes in $\mathcal{G}^{\prime} \backslash \mathcal{G}$. On cochain level for respective order complexes, it maps $c_{\mathcal{G}}^{S}$ to $c_{\mathcal{G}^{\prime}}^{S}$ for $\emptyset \neq S \subseteq[n]$. Let

$$
\begin{aligned}
& i^{\prime}:\left|\Delta\left(P_{\mathcal{G}^{\prime}} \backslash P_{\operatorname{link}(\mathcal{A})}\right)\right| \longrightarrow\left|\Gamma_{\mathcal{G}^{\prime}}\right| \backslash\left|\Gamma_{\operatorname{link}(\mathcal{A}), \mathcal{G}^{\prime}}\right|=\mathcal{M}(\mathcal{A}) \cap S^{2 d-1}, \\
& i:\left|\Delta\left(P_{\mathcal{G}} \backslash P_{\operatorname{link}(\mathcal{A})}\right)\right| \longrightarrow\left|\Gamma_{\mathcal{G}}\right| \backslash\left|\Gamma_{\operatorname{link}(\mathcal{A}), \mathcal{G}}\right|=\mathcal{M}(\mathcal{A}) \cap S^{2 d-1}
\end{aligned}
$$

denote inclusions and consider the diagram

$$
\begin{array}{cc}
\left|\Delta\left(P_{\mathcal{G}^{\prime}} \backslash P_{\operatorname{link}(\mathcal{A})}\right)\right| \stackrel{i^{\prime}}{\longrightarrow}\left|\Gamma_{\mathcal{G}^{\prime}}\right| \backslash\left|\Gamma_{\operatorname{link}(\mathcal{A}), \mathcal{G}^{\prime}}\right| \\
f \downarrow \\
\mathcal{M}(\mathcal{A}) \\
\left|\Delta\left(P_{\mathcal{G}} \backslash P_{\operatorname{link}(\mathcal{A})}\right)\right| \stackrel{i}{\longrightarrow}\left|\Gamma_{\mathcal{G}}\right| \backslash\left|\Gamma_{\operatorname{link}(\mathcal{A}), \mathcal{G}}\right| .
\end{array}
$$

We will show that this diagram commutes up to homotopy. For this, define a carrier $C$ from $\left|\Delta\left(P_{\mathcal{G}^{\prime}} \backslash P_{\operatorname{link}(\mathcal{A})}\right)\right|$ into $\mathcal{M}(\mathcal{A})$ by

$$
C\left(\theta^{(0)}<\ldots<\theta^{(d)}\right)=\overline{f\left(\theta^{(d)}\right)}
$$

for chains of cells $\theta^{(0)}<\ldots<\theta^{(d)}$ in $\Gamma_{\mathcal{G}^{\prime}} \backslash \Gamma_{\operatorname{link}(\mathcal{A})}$, where $\overline{f\left(\theta^{(d)}\right)}$ denotes the closure of the cell $f\left(\theta^{(d)}\right)$ in $\Gamma_{\mathcal{G}} \backslash \Gamma_{\operatorname{link}(\mathcal{A})}$. The inclusion $i^{\prime}$ is carried by $C$ since

$$
i^{\prime}\left(\left|\theta^{(0)}<\ldots<\theta^{(d)}\right|\right) \subseteq \overline{\theta^{(d)}} \subseteq \overline{f\left(\theta^{(d)}\right)}
$$

for $\theta^{(0)}<\ldots<\theta^{(d)}$ in $\Gamma_{\mathcal{G}^{\prime}} \backslash \Gamma_{\operatorname{link}(\mathcal{A})}$. Here $f\left(\theta^{(d)}\right)$ is the cell of lowest dimension in $\Gamma_{\mathcal{G}}$ that contains $\theta^{(d)}$ in its subdivision induced by the hyperplanes in $\mathcal{G}^{\prime} \backslash \mathcal{G}$. Also, $i \circ f$ is carried by $C$ since obviously

$$
i\left(\left|f\left(\theta^{(0)}\right)<\ldots<f\left(\theta^{(d)}\right)\right|\right) \subseteq \overline{f\left(\theta^{(d)}\right)}
$$

for $\theta^{(0)}<\ldots<\theta^{(d)}$ in $\Gamma_{\mathcal{G}^{\prime}} \backslash \Gamma_{\operatorname{link}(\mathcal{A})}$.

The Carrier Lemma [Mu, §13], $\operatorname{Bj2}$ (10.1)] implies that $i^{\prime} \sim i f$. In particular, $i^{\prime *}=f^{*} i^{*}$, and for the retractions, which are cohomology inverses of the inclusions, we have $r^{*}=r^{*} f^{*}$. We conclude that $r^{*}\left(\left[c_{\mathcal{G}^{\prime}}^{S}\right]\right)=r^{*} f^{*}\left(\left[c_{\mathcal{G}}^{S}\right]\right)=r^{*}\left(\left[c_{\mathcal{G}}^{S}\right]\right)$ for $\emptyset \neq S \subseteq[n]$.

We will see later that for any boolean arrangement the proposed cocycles $c^{S}$, $\emptyset \neq S \subseteq[n]$, represent a linear basis for the cohomology of the complement (cf. Theorem 5.2 in the broader context of geometric arrangements). Here we verify this claim for the smallest instance, an arrangement given by one single subspace in $\mathbb{C}^{d}$. The proof isolates the geometric essence of our investigations and justifies our definition of the cochains $c^{S}$ via ascending chains.

Proposition 4.6. Let $U$ be a complex subspace of codimension $m$ in $\mathbb{C}^{d}$, and let $\mathcal{G}$ be a complex hyperplane arrangement in $\mathbb{C}^{d}$ that contains a (strong) complex frame $\mathcal{H}=\left\{H_{1}, \ldots, H_{m}\right\}$ for $U$. Then, the cocycle $c^{U}:=c^{\{1\}}$ defined on $\Delta\left(P_{\mathcal{G}} \backslash P_{\operatorname{link}(\{U\})}\right)$ according to Definition 4.1 represents a generating cohomology class for $\widetilde{H}^{*}(\mathcal{M}(\{U\}))$. 
Proof. In view of Proposition 4.5 we may assume that $\mathcal{G}$ contains a complex frame for the orthogonal complement $U^{\perp}$ of $U$ in $\mathbb{C}^{d}$. The cells of $\Gamma_{\mathcal{G}}$ that lie in $U^{\perp}$ form a subcomplex $\Gamma_{\mathcal{G}_{\lceil U \perp}}$ in $\Gamma_{\mathcal{G}}$. In particular, $\left|\Gamma_{\mathcal{G}_{\left\lceil U^{\perp}\right.}}\right|$ is a deformation retract of $\left|\Gamma_{\mathcal{G}}\right| \backslash\left|\Gamma_{\operatorname{link}(\{U\})}\right|$, and the order complex of its face poset $\Delta\left(P_{\mathcal{G}_{\lceil U \perp}}\right)$ is a simplicial model for the complement of $U$ in $\mathbb{C}^{d}$.

Likewise, $\Delta\left(P_{\mathcal{G}_{U^{\perp}}}\right)$ is a simplicial model for the complement of the 0 -subspace in $U^{\perp}$ that is induced by the restriction of the arrangement $\mathcal{G}$ to $U^{\perp}$. Referring again to Proposition 4.5 we work with the simplicial model $\Delta\left(P_{\mathcal{H}_{\left\ulcorner U^{\perp}\right.}}\right)$ obtained by coarsening the stratification of $U^{\perp}$ to the one induced by the complex frame $\mathcal{H}_{\Gamma U^{\perp}}$ for $\{0\}$ in $U^{\perp}$. It follows from the proof of Proposition 4.5 that an isomorphism between the cohomology algebras of these simplicial models is induced by the poset map $f: P_{\mathcal{G}_{\Gamma U^{\perp}}} \longrightarrow P_{\mathcal{H}_{\left\lceil U^{\perp}\right.}}$, where $f$ is defined on sign vectors of cells in $\Gamma_{\mathcal{G}_{\left\lceil U^{\perp}\right.}}$ by "forgetting" the sign entries that correspond to hyperplanes in $\mathcal{G}_{\left\lceil U^{\perp}\right.} \backslash \mathcal{H}_{\left\lceil U^{\perp}\right.}$.

Since $\mathcal{H}_{\left\lceil U^{\perp}\right.}$ is a boolean hyperplane arrangement, the face poset $P_{\mathcal{H}_{\left\lceil U^{\perp}\right.}}$ of $\Gamma_{\mathcal{H}_{\left\lceil U^{\perp}\right.}}$ equals $\{0,+,-, i, j\}^{m} \backslash\{\hat{0}\}$, where $\{0,+,-, i, j\}$ has the partial order given in Section 2. The order complex $\Delta\left(P_{\mathcal{H}_{C U} \perp}\right)$ is homeomorphic to a sphere; hence, any elementary cochain that corresponds to a maximal chain in $P_{\mathcal{H}_{\lceil U} \perp}$ represents a generating cohomology class in $\widetilde{H}^{*}\left(\Delta\left(P_{\mathcal{H}_{\lceil U} \perp}\right)\right)$. We choose the maximal chain $\theta$ which is elementary ascending along the sequence $\left(H_{1} \cap U^{\perp}, \ldots, H_{m} \cap U^{\perp}\right)$ and work with $\left[\theta^{*}\right]$ as a generator for $\widetilde{H}^{*}\left(\Delta\left(P_{\mathcal{H}_{\ulcorner U \perp}}\right)\right)$. The isomorphism $f^{*}$ maps $\left[\theta^{*}\right]$ to a generator of $\widetilde{H}^{*}\left(\Delta\left(P_{\mathcal{G}_{\lceil U} \perp}\right)\right)$. In terms of elementary cochains, $f^{\sharp}\left(\theta^{*}\right)=\sum_{\tau} \tau^{*}$, where the sum is over all $2 m$-element chains of cells in $\Gamma_{\mathcal{G}_{\lceil U \perp}}$ which are elementary ascending along $\left(H_{1} \cap U^{\perp}, \ldots, H_{m} \cap U^{\perp}\right)$.

We finally get back to our initial simplicial model $\Delta\left(P_{\mathcal{G}} \backslash P_{\operatorname{link}(\{U\})}\right)$ for the complement of $U$ : The inclusion of face posets $i: P_{\mathcal{G}_{\lceil U \perp}} \longrightarrow P_{\mathcal{G}} \backslash P_{\operatorname{link}(\{U\})}$ induces an isomorphism between the cohomology algebras of the respective order complexes. Any simplicial cocycle on $\Delta\left(P_{\mathcal{G}} \backslash P_{\operatorname{link}(\{U\})}\right)$ that restricts to $f^{\sharp}\left(\theta^{*}\right)$ on $\Delta\left(P_{\mathcal{G}_{\lceil U} \perp}\right)$ represents a generating cohomology class in $\widetilde{H}^{*}\left(\Delta\left(P_{\mathcal{G}} \backslash P_{\operatorname{link}(\{U\})}\right)\right)$. Comparing explicit descriptions in terms of elementary cochains, $c^{U}$ restricts to $f^{\sharp}\left(\theta^{*}\right)$ and hence represents a generating cohomology class in $\widetilde{H}^{*}(\mathcal{M}(\{U\}))$.

Remark 4.7. The preceding proof shows that for defining a generating cocycle of a subspace complement, i.e., a generator for $\widetilde{H}^{*}(\mathcal{M}(\{U\}))$, in the spirit of Definition 4.1 any "dense" ascending pattern of sign vector entries with respect to $\left(H_{1}, \ldots, H_{m}\right)$ could be used. Our convention is compatible with that of [BZ] for the hyperplane case.

\section{Linear bases For the COHOMOLOGY OF GEOMETRIC ARRANGEMENTS}

Now we extend our investigations to complex subspace arrangements which have a geometric intersection lattice. First we comment on the choice of appropriate combinatorial stratifications. Working with the induced simplicial models for arrangement complements, we transfer the simplicial cocycles introduced in Section 4 to geometric arrangements by restriction from the complements of boolean subarrangements. Among the cocycles thus obtained we distinguish the representatives of a linear basis for the cohomology of a geometric arrangement. 
In order to fix combinatorial terminology, recall that a finite lattice $\mathcal{L}$ is geometric if it is semimodular and all elements are joins of atoms (elements of rank 1) [CR, Chap. 2] $\mathrm{Ox}$, Sect. 1.7]. For a set of atoms $S$ in $\mathcal{L}, \operatorname{rank}(\bigvee S) \leq|S| ; S$ is independent if equality holds, otherwise it is dependent. A maximal independent set is a basis of $\mathcal{L}$; a minimal dependent set is a circuit. The collection of all non-empty independent sets forms a simplicial complex — the matroid complex $\mathcal{I}(\mathcal{L})$ Bj1. Sect. 7.3]. We denote the collection of its facets, the maximal independent sets in $\mathcal{L}$, by $\mathcal{B}(\mathcal{L})$. Assume that the atoms in $\mathcal{L}$ are given a linear order. A circuit in $\mathcal{L}$ minus its smallest element is called a broken circuit. The subcomplex of $\mathcal{I}(\mathcal{L})$ formed by all non-empty sets of atoms that do not contain a broken circuit is called the broken circuit complex $\mathrm{BC}(\mathcal{L})$. See $\mathrm{Bj} 1, \mathrm{Bry}$ for additional information.

Let $\mathcal{A}=\left\{U_{1}, \ldots, U_{n}\right\}$ be an arrangement of complex subspaces in $\mathbb{C}^{d}$ with geometric intersection lattice $\mathcal{L}=\mathcal{L}(\mathcal{A})$ - we call $\mathcal{A}$ a geometric arrangement for brevity. We identify the atoms in $\mathcal{L}$ with the elements of the index set of subspaces in $\mathcal{A}$, and thus define the subarrangement $\mathcal{A}_{S}:=\left\{U_{i}\right\}_{i \in S}$ for any set of atoms $S$ in $\mathcal{L}$. Observe that $\mathcal{A}_{S}$ is itself a geometric arrangement with intersection lattice $\mathcal{L}\left(\mathcal{A}_{S}\right)$, the join sublattice of $\mathcal{L}$ generated by the atoms contained in $S$. Moreover, $\mathcal{A}_{S}$ is boolean if and only if the index set $S$ is independent in $\mathcal{L}$.

To obtain a combinatorial stratification of $\mathbb{C}^{d}$ that is suitable for the study of the arrangement $\mathcal{A}$, choose a framing arrangement $\mathcal{A}_{B, \triangle}$ and a strong complex frame $\widetilde{\mathcal{H}}\left(\mathcal{A}_{B}\right)$ for each maximal boolean subarrangement $\mathcal{A}_{B}, B \in \mathcal{B}(\mathcal{L})$. Fix a hyperplane arrangement $\mathcal{G}$ in $\mathbb{C}^{d}$ that contains the strong complex frames $\widetilde{\mathcal{H}}\left(\mathcal{A}_{B}\right)$ for all $B \in \mathcal{B}(\mathcal{L})$. In the sequel, we work with the induced simplicial model $\Delta\left(P_{\mathcal{G}} \backslash P_{\operatorname{link}(\mathcal{A})}\right)$ for the complement of $\mathcal{A}$.

Definition 5.1. Let $\mathcal{A}=\left\{U_{1}, \ldots, U_{n}\right\}$ be an arrangement of complex subspaces in $\mathbb{C}^{d}$ with geometric intersection lattice $\mathcal{L}=\mathcal{L}(\mathcal{A})$. Assume that the set of bases $\mathcal{B}(\mathcal{L})$ is linearly ordered by reversed lexicographic order. For $S=\left\{i_{1}, \ldots, i_{k}\right\}<\in \mathcal{I}(\mathcal{L})$, let $B(S)$ be the minimal basis in $\mathcal{L}$ that contains $S$, and let $c_{\mathcal{A}_{B(S)}}^{S}$ denote the cocycle defined on the simplicial model $\Delta\left(P_{\mathcal{G}} \backslash P_{\operatorname{link}\left(\mathcal{A}_{B(S)}\right)}\right)$ for $\mathcal{M}\left(\mathcal{A}_{B(S)}\right)$ according to Definition 4.1. Define a cocycle $c^{S} \in C^{2 \operatorname{codim}_{\mathbb{C}} U_{S}-|S|}\left(\Delta\left(P_{\mathcal{G}} \backslash P_{\operatorname{link}(\mathcal{A})}\right)\right)$ by restriction of the cocycle $c_{\mathcal{A}_{B(S)}^{S}}^{S}$ to $\Delta\left(P_{\mathcal{G}} \backslash P_{\operatorname{link}(\mathcal{A})}\right)$,

$$
c^{S}:=i^{\sharp}\left(c_{\mathcal{A}_{B(S)}^{S}}^{S},\right.
$$

where $i: \Delta\left(P_{\mathcal{G}} \backslash P_{\operatorname{link}(\mathcal{A})}\right) \longrightarrow \Delta\left(P_{\mathcal{G}} \backslash P_{\operatorname{link}\left(\mathcal{A}_{B(S)}\right)}\right)$ denotes the natural inclusion.

As a restriction of a cocycle, the cochain $c^{S}$ is a cocycle by definition. In terms of elementary cochains, it can be written as $c^{S}=\varepsilon_{|S|} \sum_{\tau} \tau^{*}$, where the sum is over all chains of cells $\tau$ in $\Gamma_{\mathcal{G}} \backslash \Gamma_{\operatorname{link}(\mathcal{A})}$ that are ascending along $\left(\widetilde{\mathcal{F}}_{i_{1}}^{S}\left(\mathcal{A}_{B(S)}\right), \ldots\right.$, $\left.\widetilde{\mathcal{F}}_{i_{k}}^{S}\left(\mathcal{A}_{B(S)}\right)\right)$ - the characteristic sequences of hyperplanes chosen from the strong complex frame $\widetilde{\mathcal{H}}\left(\mathcal{A}_{B(S)}\right)$ for the boolean subarrangement $\mathcal{A}_{B(S)}$ of $\mathcal{A}$.

Theorem 5.2. Let $\mathcal{A}=\left\{U_{1}, \ldots, U_{n}\right\}$ be an arrangement of complex subspaces in $\mathbb{C}^{d}$ with geometric intersection lattice $\mathcal{L}=\mathcal{L}(\mathcal{A})$. Then the set of cohomology classes

$$
\left\{\left[c^{S}\right]: S \in \mathrm{BC}(\mathcal{L})\right\}
$$

is a $\mathbb{Z}$-linear basis for the reduced cohomology of the complement of $\mathcal{A}$. 
Proof. The proof is by induction on the number of subspaces in $\mathcal{A}$. The induction start is covered by Proposition 4.6 where we verified that $\left[c^{\{1\}}\right]$ is a cohomology generator for an arrangement $\mathcal{A}=\left\{U_{1}\right\}$ consisting of one single subspace.

For the induction step, let $\mathcal{A}=\left\{U_{1}, \ldots, U_{n}\right\}$ be a geometric arrangement in $\mathbb{C}^{d}$ on $n$ subspaces with intersection lattice $\mathcal{L}=\mathcal{L}(\mathcal{A})$. Both the deletion $\mathcal{A}^{\prime}:=\mathcal{A} \backslash\left\{U_{n}\right\}$ and the restriction $\mathcal{A}^{\prime \prime}=\left\{U_{1} \cap U_{n}, \ldots, U_{n-1} \cap U_{n}\right\}$ are geometric arrangements. Their intersection lattices are $\mathcal{L}^{\prime}:=\mathcal{L}\left(\mathcal{A}^{\prime}\right)$, the join sublattice generated by the atoms $1, \ldots, n-1$ in $\mathcal{L}(\mathcal{A})$, and $\mathcal{L}^{\prime \prime}:=\mathcal{L}\left(\mathcal{A}^{\prime \prime}\right)$, the interval $\left[U_{n}, \hat{1}\right]$ in $\mathcal{L}(\mathcal{A})$. Observe that $\mathcal{A}^{\prime}$ is an arrangement of exactly $n-1$ subspaces, whereas $\mathcal{A}^{\prime \prime}$ is an arrangement of at most $n-1$ subspaces.

On the combinatorial side, we use the recursive construction of the broken circuit complex of $\mathcal{L}$ from the broken circuit complexes of $\mathcal{L}^{\prime}$ and $\mathcal{L}^{\prime \prime}$ due to Brylawski [Bry]:

$$
\mathrm{BC}(\mathcal{L})=\mathrm{BC}\left(\mathcal{L}^{\prime}\right) \cup\{\{n\}\} \cup\left\{S \cup\{n\}: S \in \mathrm{BC}\left(\mathcal{L}^{\prime \prime}\right)\right\} .
$$

On the topological side, our inductive proof relies on a cohomological Mayer-Vietoris sequence that involves the complements of the arrangement $\mathcal{A}$, of its deletion $\mathcal{A}^{\prime}$, of its restriction $\mathcal{A}^{\prime \prime}$, and of a single subspace. We view the complement of $\mathcal{A}$ as the intersection of the complement of $\mathcal{A}^{\prime}$ with the complement of the single subspace $U_{n}$,

$$
\mathcal{M}(\mathcal{A})=\mathcal{M}\left(\mathcal{A}^{\prime}\right) \cap \mathcal{M}\left(\left\{U_{n}\right\}\right) .
$$

As the union of these spaces we obtain the complement of the restriction $\mathcal{A}^{\prime \prime}$,

$$
\mathcal{M}\left(\mathcal{A}^{\prime}\right) \cup \mathcal{M}\left(\left\{U_{n}\right\}\right)=\mathcal{M}\left(\mathcal{A}^{\prime \prime}\right) .
$$

The Mayer-Vietoris sequence in reduced cohomology [Br, Ch.V, Thm. 8.3] for the union of the spaces $\mathcal{M}\left(\mathcal{A}^{\prime}\right)$ and $\mathcal{M}\left(\left\{U_{n}\right\}\right)$ is

$$
\stackrel{\left(i_{3}^{*}, i_{4}^{*}\right)}{\longrightarrow} \widetilde{H}^{t}\left(\mathcal{M}\left(\mathcal{A}^{\prime}\right)\right) \oplus \widetilde{H}^{t}\left(\mathcal{M}\left(\left\{U_{n}\right\}\right)\right) \stackrel{i_{1}^{*}-i_{2}^{*}}{\longrightarrow} \widetilde{H}^{t}(\mathcal{M}(\mathcal{A})) \stackrel{\delta^{*}}{\longrightarrow} \widetilde{H}^{t+1}\left(\mathcal{M}\left(\mathcal{A}^{\prime \prime}\right)\right) \stackrel{\left(i_{3}^{*}, i_{4}^{*}\right)}{\longrightarrow}
$$

where the maps $i_{j}^{*}, j=1, \ldots, 4$, are induced by inclusions.

We have to make sure that the stratification of $\mathbb{C}^{d}$ by the hyperplane arrangement $\mathcal{G}$ that we chose with respect to the arrangement $\mathcal{A}$ is also suitable for the study of the arrangements $\mathcal{A}^{\prime}$ and $\mathcal{A}^{\prime \prime}$ :

Lemma 5.3. For a geometric arrangement $\mathcal{A}=\left\{U_{1}, \ldots, U_{n}\right\}$ in $\mathbb{C}^{d}$, let $\mathcal{G}$ be a hyperplane arrangement that contains a strong complex frame $\widetilde{\mathcal{H}}\left(\mathcal{A}_{B}\right)$ for each maximal boolean subarrangement $\mathcal{A}_{B}$ in $\mathcal{A}, B \in \mathcal{B}(\mathcal{L})$. Then $\mathcal{G}$ contains strong complex frames for all maximal boolean subarrangements of both the deletion and of the restriction of $\mathcal{A}$.

Proof. Maximal boolean subarrangements of the deletion $\mathcal{A}^{\prime}$ are either maximal in $\mathcal{A}$ or they are deletions of maximal boolean subarrangements in $\mathcal{A}$. Maximal boolean subarrangements in the restriction $\mathcal{A}^{\prime \prime}$ are restrictions of maximal boolean subarrangements in $\mathcal{A}$. By our discussion in Section 3 the hyperplane arrangement $\mathcal{G}$ thus contains the needed strong frames.

Thus, $\mathcal{G}$ simultaneously induces simplicial models for the complements of the geometric arrangements $\mathcal{A}, \mathcal{A}^{\prime}$ and $\mathcal{A}^{\prime \prime}$, and also for the arrangement consisting of the single subspace $U_{n}$. We will use the same notation for inclusions between these simplicial models as introduced above for the inclusions between the actual complements.

By induction, the cocycles $c_{\mathcal{A}^{\prime}}^{S} \in C^{*}\left(\Delta\left(P_{\mathcal{G}} \backslash P_{\operatorname{link}\left(\mathcal{A}^{\prime}\right)}\right)\right)$ for $S \in \mathrm{BC}\left(\mathcal{L}^{\prime}\right)$ represent a linear basis for $\widetilde{H}^{*}\left(\mathcal{M}\left(\mathcal{A}^{\prime}\right)\right)$. The characteristic sequences of hyperplanes that occur 
in the description of $c_{\mathcal{A}^{\prime}}^{S}$ in terms of elementary cochains are chosen from the strong complex frame $\widetilde{\mathcal{H}}\left(\mathcal{A}_{B_{\mathcal{L}^{\prime}}(S)}^{\prime}\right)$ for the maximal boolean subarrangement $\mathcal{A}_{B_{\mathcal{L}^{\prime}}(S)}^{\prime}$ in $\mathcal{A}^{\prime}$. Recall that $B_{\mathcal{L}^{\prime}}(S)$ denotes the reversed lexicographically minimal basis in $\mathcal{L}^{\prime}$ that contains $S$.

Consider $S$ as an element in $\operatorname{BC}(\mathcal{L})$. If $\operatorname{rank} \mathcal{L}=\operatorname{rank} \mathcal{L}^{\prime}$, any basis of $\mathcal{L}$ that is not a basis of $\mathcal{L}^{\prime}$ contains $n$ and hence is reversed lexicographically larger than $B_{\mathcal{L}^{\prime}}(S)$. We conclude that $B_{\mathcal{L}}(S)=B_{\mathcal{L}^{\prime}}(S)$. If $\operatorname{rank} \mathcal{L}<\operatorname{rank} \mathcal{L}^{\prime}$, any basis of $\mathcal{L}$ contains $n$ and their linear order is determined by elements from $[n-1]$, hence $B_{\mathcal{L}}(S)=B_{\mathcal{L}^{\prime}}(S) \cup\{n\}$.

Thus, $\widetilde{\mathcal{H}}\left(\mathcal{A}_{B_{\mathcal{L}^{\prime}}(S)}^{\prime}\right)$ either coincides with $\widetilde{\mathcal{H}}\left(\mathcal{A}_{B_{\mathcal{L}}(S)}\right)$ or it is the deletion of $\widetilde{\mathcal{H}}\left(\mathcal{A}_{B_{\mathcal{L}}(S)}\right)$. We refer the latter case to Proposition 3.5 $(i)$, and conclude that the characteristic sequences of hyperplanes for $S$ in $\mathcal{A}^{\prime}$ coincide with those for $S$ in $\mathcal{A}$. We obtain $c_{\mathcal{A}}^{S}$ from $c_{\mathcal{A}^{\prime}}^{S}$ by restriction, as one sees from the explicit description of these cochains:

$$
i_{1}^{\sharp}\left(c_{\mathcal{A}^{\prime}}^{S}\right)=c_{\mathcal{A}}^{S} \quad \text { for } S \in \operatorname{BC}\left(\mathcal{L}^{\prime}\right) .
$$

We define a cocycle $c^{U_{n}}$ on $\Delta\left(P_{\mathcal{G}} \backslash P_{\operatorname{link}\left(\left\{U_{n}\right\}\right)}\right)$ that represents a generator for $\widetilde{H}^{*}\left(\mathcal{M}\left(\left\{U_{n}\right\}\right)\right)$ (cf. Proposition 4.6) with respect to the strong complex frame used for the definition of $c_{\mathcal{A}}^{\{n\}}$ on $\Delta\left(P_{\mathcal{G}} \backslash P_{\operatorname{link}(\mathcal{A})}\right)$. Thus, $c_{\mathcal{A}}^{\{n\}}$ is obtained from $c^{U_{n}}$ by restriction:

$$
i_{2}^{\sharp}\left(c^{U_{n}}\right)=c_{\mathcal{A}}^{\{n\}} .
$$

By induction, the cocycles $c_{\mathcal{A}^{\prime \prime}}^{S} \in C^{*}\left(\Delta\left(P_{\mathcal{G}} \backslash P_{\operatorname{link}\left(\mathcal{A}^{\prime \prime}\right)}\right)\right)$ for $S \in \mathrm{BC}\left(\mathcal{L}^{\prime \prime}\right)$ represent a linear basis for $\widetilde{H}^{*}\left(\mathcal{M}\left(\mathcal{A}^{\prime \prime}\right)\right)$. The characteristic sequences of hyperplanes for $S$ in $\mathcal{A}^{\prime \prime}$ are chosen from the strong complex frame $\widetilde{\mathcal{H}}\left(\mathcal{A}_{B_{\mathcal{L}^{\prime \prime}}(S)}^{\prime \prime}\right)$ for the maximal boolean subarrangement $\mathcal{A}_{B_{\mathcal{L}^{\prime \prime}}(S)}^{\prime \prime}$ in $\mathcal{A}^{\prime \prime}$. With $S \cup\{n\} \in \mathrm{BC}(\mathcal{L})$ according to $(*)$, we can compare minimal bases in $\mathcal{L}^{\prime \prime}$, resp. $\mathcal{L}$ that contain $S$, resp. $S \cup\{n\}$ : Since all bases in $\mathcal{L}$ that contain $S \cup\{n\}$ in particular contain $n$, their order is determined by elements in $[n-1]$, hence $B_{\mathcal{L}}(S \cup\{n\})=B_{\mathcal{L}^{\prime \prime}}(S) \cup\{n\}$. Thus, $\mathcal{A}_{B_{\mathcal{L}^{\prime \prime}}(S)}^{\prime \prime}$ is the restriction of the boolean subarrangement $\mathcal{A}_{B_{\mathcal{L}}(S \cup\{n\})}$ in $\mathcal{A}$. We conclude that $\widetilde{\mathcal{H}}\left(\mathcal{A}_{B_{\mathcal{L}^{\prime \prime}}(S)}^{\prime \prime}\right)$ is the restriction of the strong complex frame $\widetilde{\mathcal{H}}\left(\mathcal{A}_{B_{\mathcal{L}}(S \cup\{n\})}\right)$ from which characteristic sequences of hyperplanes are chosen for the definition of $c_{\mathcal{A}}^{S \cup\{n\}}$.

Here is the crucial step of our proof.

Lemma 5.4. The connecting homomorphism in the cohomological Mayer-Vietoris sequence for $\mathcal{M}\left(\mathcal{A}^{\prime}\right) \cup \mathcal{M}\left(\left\{U_{n}\right\}\right)$ is surjective,

$$
\delta^{*}\left(\left[c_{\mathcal{A}}^{S \cup\{n\}}\right]\right)=\left[c_{\mathcal{A}^{\prime \prime}}^{S}\right] \quad \text { for } S \in \mathrm{BC}\left(\mathcal{L}^{\prime \prime}\right) .
$$

This lemma implies that the Mayer-Vietoris sequence decomposes into short exact sequences:

$$
0 \longrightarrow \widetilde{H}^{t}\left(\mathcal{M}\left(\mathcal{A}^{\prime}\right)\right) \oplus \widetilde{H}^{t}\left(\mathcal{M}\left(\left\{U_{n}\right\}\right)\right) \longrightarrow \widetilde{H}^{t}(\mathcal{M}(\mathcal{A})) \stackrel{\delta^{*}}{\longrightarrow} \widetilde{H}^{t+1}\left(\mathcal{M}\left(\mathcal{A}^{\prime \prime}\right)\right) \longrightarrow 0 .
$$


Moreover, $\widetilde{H}^{*}\left(\mathcal{M}\left(\mathcal{A}^{\prime \prime}\right)\right)$ is free abelian by induction hypothesis, hence the sequences split. In particular, a linear basis for $\widetilde{H}^{*}(\mathcal{M}(\mathcal{A}))$ is represented by the cocycles

$$
\begin{array}{ll}
c_{\mathcal{A}}^{S}=i_{1}^{\sharp}\left(c_{\mathcal{A}^{\prime}}^{S}\right) & \text { for } S \in \operatorname{BC}\left(\mathcal{L}^{\prime}\right), \\
c_{\mathcal{A}}^{\{n\}}=i_{2}^{\sharp}\left(c^{U_{n}}\right), & \\
c_{\mathcal{A}}^{S \cup\{n\}} \in\left(\delta^{*}\right)^{-1}\left(\left[c_{\mathcal{A}^{\prime \prime}}^{S}\right]\right) & \text { for } S \in \operatorname{BC}\left(\mathcal{L}^{\prime \prime}\right) .
\end{array}
$$

Observe that the index sets combine to the broken circuit complex of $\mathcal{L}$ by its recursive construction $(*)$, thus the above list proves Theorem 5.2

Proof of Lemma 5.4. For $S=\left\{i_{1}, \ldots, i_{k}\right\}_{<} \in \mathrm{BC}\left(\mathcal{L}^{\prime \prime}\right)$ consider the boolean subarrangement $\mathcal{B}:=\mathcal{A}_{B_{\mathcal{L}}(S \cup\{n\})}$ in $\mathcal{A}$. We study the cohomological MayerVietoris sequence for the union of the spaces $\mathcal{M}\left(\mathcal{B}^{\prime}\right)$ and $\mathcal{M}\left(\left\{U_{n}\right\}\right)$. The inclusion $i: \mathcal{M}\left(\mathcal{A}^{\prime \prime}\right) \longrightarrow \mathcal{M}\left(\mathcal{B}^{\prime \prime}\right)$ induces a map of Mayer-Vietoris sequences:

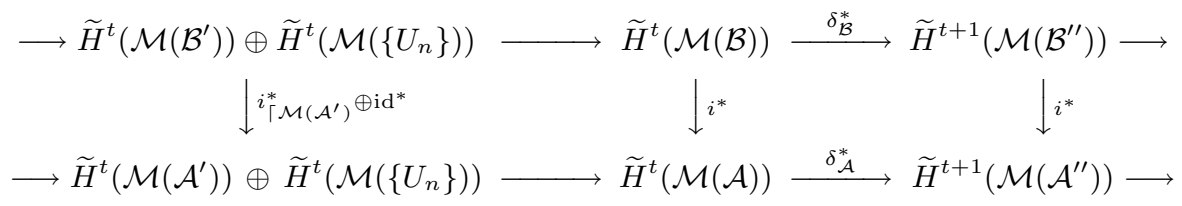

Let $c_{\mathcal{B}}^{S \cup\{n\}}$ and $c_{\mathcal{B}^{\prime \prime}}^{S}$ denote the standard cocycles on the simplicial models $\Delta\left(P_{\mathcal{G}} \backslash P_{\operatorname{link}(\mathcal{B})}\right)$, resp. $\Delta\left(P_{\mathcal{G}} \backslash P_{\operatorname{link}\left(\mathcal{B}^{\prime \prime}\right)}\right)$, defined with respect to the strong complex frame $\widetilde{\mathcal{H}}(\mathcal{B})=\widetilde{\mathcal{H}}\left(\mathcal{A}_{B_{\mathcal{L}}(S \cup\{n\})}\right)$, resp. its restriction $\widetilde{\mathcal{H}}^{\prime \prime}(\mathcal{B})$. As discussed above, $c_{\mathcal{A}}^{S \cup\{n\}}$ and $c_{\mathcal{A}^{\prime \prime}}^{S}$ are defined with respect to these strong complex frames as well. By definition,

$$
\left[c_{\mathcal{A}}^{S \cup\{n\}}\right]=i^{*}\left(\left[c_{\mathcal{B}}^{S \cup\{n\}}\right]\right) \quad \text { and } \quad\left[c_{\mathcal{A}^{\prime \prime}}^{S}\right]=i^{*}\left(\left[c_{\mathcal{B}^{\prime \prime}}^{S}\right]\right) .
$$

It suffices to show that

$$
\delta_{\mathcal{B}}^{*}\left(\left[c_{\mathcal{B}}^{S \cup\{n\}}\right]\right)=\left[c_{\mathcal{B}^{\prime \prime}}^{S}\right]
$$

by naturality of the Mayer-Vietoris sequence we then conclude that

$$
\delta_{\mathcal{A}}^{*}\left(\left[c_{\mathcal{A}}^{S \cup\{n\}}\right]\right)=\delta_{\mathcal{A}}^{*} i^{*}\left(\left[c_{\mathcal{B}}^{S \cup\{n\}}\right]\right)=i^{*} \delta_{\mathcal{B}}^{*}\left(\left[c_{\mathcal{B}}^{S \cup\{n\}}\right]\right)=i^{*}\left(\left[c_{\mathcal{B}^{\prime \prime}}^{S}\right]\right)=\left[c_{\mathcal{A}^{\prime \prime}}^{S}\right] .
$$

Thus we concentrate on the connecting homomorphism $\delta_{\mathcal{B}}^{*}$ in the Mayer-Vietoris sequence for the boolean arrangement $\mathcal{B}$. Now all the cocycles involved are defined with respect to one single strong complex frame, namely $\widetilde{\mathcal{H}}(\mathcal{B})$. The connecting homomorphism $\delta_{\mathcal{B}}^{*}: \widetilde{H}^{*}(\mathcal{M}(\mathcal{B})) \longrightarrow \widetilde{H}^{*+1}\left(\mathcal{M}\left(\mathcal{B}^{\prime \prime}\right)\right)$ can be described as

$$
\delta_{\mathcal{B}}^{*}([c])=\left[\left(i_{3}^{\sharp}, i_{4}^{\sharp}\right)^{-1} \circ \delta \circ\left(i_{1}^{\sharp}-i_{2}^{\sharp}\right)^{-1}(c)\right] \quad \text { for }[c] \in \widetilde{H}^{*}(\mathcal{M}(\mathcal{B})),
$$

resulting from the following diagram of cochain complexes with exact rows:

$$
\begin{array}{cc}
C^{t+1}\left(\mathcal{M}\left(\mathcal{B}^{\prime \prime}\right)\right) \stackrel{\left(i_{3}^{\sharp}, i_{4}^{\sharp}\right)}{\longrightarrow} C^{t+1}\left(\mathcal{M}\left(\mathcal{B}^{\prime}\right)\right) \oplus C^{t+1}\left(\mathcal{M}\left(\left\{U_{n}\right\}\right)\right) & \longrightarrow \\
\uparrow \delta & \ldots \\
\ldots \quad & \longrightarrow C^{t}\left(\mathcal{M}\left(\mathcal{B}^{\prime}\right)\right) \oplus C^{t}\left(\mathcal{M}\left(\left\{U_{n}\right\}\right)\right) \stackrel{\left(i_{1}^{\sharp}-i_{2}^{\sharp}\right)}{\longrightarrow} C^{t}(\mathcal{M}(\mathcal{B})) .
\end{array}
$$

We sketch how to trace the representing cocycle $c_{\mathcal{B}}^{S \cup\{n\}} \in C^{*}(\mathcal{M}(\mathcal{B}))$ through this diagram. Details can be found in Fe, Thm. 1.5.2]. 
Recall the description of $c_{\mathcal{B}}^{S \cup\{n\}}$ as a sum of elementary cochains:

$$
c_{\mathcal{B}}^{S \cup\{n\}}=\varepsilon_{|S|+1} \sum_{\tau} \tau^{*},
$$

where the sum is over all $\left(l_{S}+1\right)$-element chains, $l_{S}:=2 \operatorname{codim}_{\mathbb{C}} U_{S \cup\{n\}}-|S|-1$, in $\Gamma_{\mathcal{G}} \backslash \Gamma_{\operatorname{link}(\mathcal{B})}$ which are ascending along

$$
\left(\widetilde{\mathcal{F}}_{i_{1}}^{S \cup\{n\}}(\mathcal{B}), \ldots, \tilde{\mathcal{F}}_{i_{k}}^{S \cup\{n\}}(\mathcal{B}), \widetilde{\mathcal{F}}_{n}^{S \cup\{n\}}(\mathcal{B})\right) .
$$

The cocycle $c_{\mathcal{B}^{\prime \prime}}^{S}$ can be described as $c_{\mathcal{B}^{\prime \prime}}^{S}=\varepsilon_{|S|} \sum_{\tau} \tau^{*}$, where the sum is over all $\left(l_{S}+2\right)$-element chains of cells in $\Gamma_{\mathcal{G}} \backslash \Gamma_{\operatorname{link}\left(\mathcal{B}^{\prime \prime}\right)}$ that are ascending along $\left(\widetilde{\mathcal{F}}_{i_{1}}^{S}\left(\mathcal{B}^{\prime \prime}\right), \ldots, \widetilde{\mathcal{F}}_{i_{k}}^{S}\left(\mathcal{B}^{\prime \prime}\right)\right)$; by Proposition 3.5 (ii) the latter sequences coincide with

$$
\left(\widetilde{\mathcal{F}}_{i_{1}}^{S \cup\{n\}}(\mathcal{B}), \ldots, \widetilde{\mathcal{F}}_{i_{k}}^{S \cup\{n\}}(\mathcal{B}) \cup \widetilde{\mathcal{F}}_{n}^{S \cup\{n\}}(\mathcal{B})\right) .
$$

Step 1. We propose a cochain $\left(\rho^{S}, 0\right) \in C^{l_{S}}\left(\mathcal{M}\left(\mathcal{B}^{\prime}\right)\right) \oplus C^{l_{S}}\left(\mathcal{M}\left(\left\{U_{n}\right\}\right)\right)$ as an inverse image of $c_{\mathcal{B}}^{S \cup\{n\}}$ under $i_{1}^{\sharp}-i_{2}^{\sharp}$ :

$$
\rho^{S}:=\varepsilon_{|S|+1} \sum_{\tau} \sum_{t=0}^{l_{S}-2 m_{n}+1} \tau_{t}^{*},
$$

where the first sum is over all $\left(l_{S}+1\right)$-element chains $\tau: \tau^{(0)}<\ldots<\tau^{\left(l_{S}\right)}$ in $\Gamma_{\mathcal{G}} \backslash \Gamma_{\operatorname{link}(\mathcal{B})}$ which are ascending along

$$
\left(\widetilde{\mathcal{F}}_{i_{1}}^{S \cup\{n\}}(\mathcal{B}), \ldots, \widetilde{\mathcal{F}}_{i_{k}}^{S \cup\{n\}}(\mathcal{B}), \widetilde{\mathcal{F}}_{n}^{S \cup\{n\}}(\mathcal{B})\right),
$$

and the chain $\tau_{t}$ is obtained from $\tau$ by altering the first $t$ cells in one sign vector coordinate: $\tau_{t}^{(j)}\left(H_{n, 0}\right)=0$ for $j=0, \ldots, t-1$. We emphasize that $\tau_{0}$ coincides with $\tau$, and that $\tau_{l_{S}-2 m_{n}+1}$ has the following sign pattern with respect to $\widetilde{\mathcal{F}}_{n}^{S \cup\{n\}}(\mathcal{B})$ :

$$
\tau_{l_{S}-2 m_{n}+1}^{(j)}\left(\tilde{\mathcal{F}}_{n}^{S \cup\{n\}}(\mathcal{B})\right)=(0, \ldots, 0) \quad \text { for } j=0, \ldots, l_{S}-2 m_{n}
$$

and on the rest of the cells, $\tau_{l_{S}-2 m_{n}+1}^{\left(l_{S}-2 m_{n}+1\right)}<\ldots<\tau_{l_{S}-2 m_{n}+1}^{\left(l_{S}\right)}$, the chain is elementary ascending along the hyperplanes in $\widetilde{\mathcal{F}}_{n}^{S \cup\{n\}}(\mathcal{B})$.

For $t>0$, there are cells in $\tau_{t}$ that are not contained in $\Gamma_{\mathcal{G}} \backslash \Gamma_{\operatorname{link}(\mathcal{B})}$; restriction maps the corresponding elementary cochains to zero. Hence $i_{1}^{\sharp}\left(\rho^{S}\right)=\varepsilon_{|S|+1} \sum_{\tau} \tau_{0}^{*}$ $=c_{\mathcal{B}}^{S \cup\{n\}}$.

Step 2. We now describe the image of $\rho^{S}$ under the coboundary operator $\delta$. We propose the following cochain in $C^{l_{S}+1}\left(\mathcal{M}\left(\mathcal{B}^{\prime}\right)\right)$ :

$$
\delta\left(\rho^{S}\right)=\varepsilon_{|S|} \sum_{\tau} \sum_{\widehat{\tau}} \hat{\tau}^{*}
$$

where the first sum is over all $\left(l_{S}+1\right)$-element chains in $\Gamma_{\mathcal{G}} \backslash \Gamma_{\operatorname{link}(\mathcal{B})}$ which are ascending along

$$
\left(\widetilde{\mathcal{F}}_{i_{1}}^{S \cup\{n\}}(\mathcal{B}), \ldots, \widetilde{\mathcal{F}}_{i_{k}}^{S \cup\{n\}}(\mathcal{B}), \widetilde{\mathcal{F}}_{n}^{S \cup\{n\}}(\mathcal{B})\right),
$$

and the second sum is over $\left(l_{S}+2\right)$-element chains $\hat{\tau}$ in $\Gamma_{\mathcal{G}} \backslash \Gamma_{\operatorname{link}\left(\mathcal{B}^{\prime}\right)}$ which are obtained from $\tau$ by altering the relative positions of the first $l_{S}-2 m_{n}+1$ cells with 
respect to the hyperplane $H_{n, 0}$, namely to $\hat{\tau}^{(j)}\left(H_{n, 0}\right)=0, j=0, \ldots, l_{S}-2 m_{n}$, and inserting a cell $\sigma$ after $\hat{\tau}^{\left(l_{S}-2 m_{n}\right)}$ with

$$
\begin{aligned}
& \sigma\left(\widetilde{\mathcal{F}}_{i_{r}}^{S \cup\{n\}}(\mathcal{B})\right)=(i, \ldots, i) \quad \text { for } r=1, \ldots, k, \\
& \sigma\left(\widetilde{\mathcal{F}}_{n}^{S \cup\{n\}}(\mathcal{B})\right)=(0, \ldots, 0) .
\end{aligned}
$$

We emphasize the two main features of chains $\hat{\tau}$ in $\Gamma_{\mathcal{G}} \backslash \Gamma_{\operatorname{link}\left(\mathcal{B}^{\prime}\right)}$ :

(i) The deletion of the cell $\hat{\tau}^{\left(l_{S}-2 m_{n}+1\right)}$ results in a chain $\tau_{l_{S}-2 m_{n}+1}$ as described in Step 1.

(ii) The chains $\hat{\tau}$ are ascending along

$$
\left(\widetilde{\mathcal{F}}_{i_{1}}^{S \cup\{n\}}(\mathcal{B}), \ldots, \widetilde{\mathcal{F}}_{i_{k}}^{S \cup\{n\}}(\mathcal{B}) \cup \widetilde{\mathcal{F}}_{n}^{S \cup\{n\}}(\mathcal{B})\right) .
$$

We leave the verification of the description for $\delta\left(\rho^{S}\right)$ to the reader. As in the proof of Proposition 4.2 one can show that non-trivial contributions in $\delta \rho^{S}(\theta)=$ $\sum_{j=0}^{l_{S}+1}(-1)^{j} \rho^{S}\left(\theta_{j}\right)$ can be paired such that they cancel, unless $\theta$ is one of the chains $\hat{\tau}$ described above. For those, $\delta \rho^{S}$ evaluates to $(-1)^{l_{S}-2 m_{n}+1} \varepsilon_{|S|+1}=\varepsilon_{|S|}$.

Step 3. We finally assert that

$$
\left(i_{3}^{\sharp}, i_{4}^{\sharp}\right)\left(c_{\mathcal{B}^{\prime \prime}}^{S}\right)=\left(\delta \rho^{S}, 0\right),
$$

which is immediately seen from the explicit descriptions of the cochains involved. Thus, $\left[c_{\mathcal{B}}^{S \cup\{n\}}\right]$ maps under $\delta_{\mathcal{B}}^{*}$ as claimed.

\section{Representatives for Generating COHOMOlOGy Classes}

Our next aim is to understand multiplication and to derive relations among the cohomology classes that are represented by the cocycles of Definition 5.1 In this section we obtain more flexibility in their construction. As a first step we analyze the simplicial model for the complement of a boolean arrangement that is induced by a hyperplane arrangement which contains different strong complex frames, and we compare the corresponding cocycles.

Proposition 6.1. Let $\mathcal{A}=\left\{U_{1}, \ldots, U_{n}\right\}$ be a boolean subspace arrangement in $\mathbb{C}^{d}$, and let $\widetilde{\mathcal{H}}^{0}$ and $\widetilde{\mathcal{H}}^{1}$ be strong complex frames for $\mathcal{A}$ with respect to framing arrangements $\mathcal{A}_{\triangle}^{0}$ and $\mathcal{A}_{\triangle}^{1}$. Let $\mathcal{G}$ be a complex hyperplane arrangement that contains both $\widetilde{\mathcal{H}}^{0}$ and $\widetilde{\mathcal{H}}^{1}$. Denote by $c_{\widetilde{\mathcal{H}}^{t}}^{S}, t=0,1$, the standard cocycles defined on $\Delta\left(P_{\mathcal{G}} \backslash P_{\operatorname{link}(\mathcal{A})}\right)$ with respect to the strong complex frame indicated by the index. Then, the induced cohomology classes coincide:

$$
\left[c_{\widetilde{\mathcal{H}}^{0}}^{S}\right]=\left[c_{\widetilde{\mathcal{H}}^{1}}^{S}\right] \quad \text { for } \emptyset \neq S \subseteq[n] .
$$

Proof. The proof is by induction on $n$.

For $n=1$ let $U$ be a complex subspace of codimension $m$ in $\mathbb{C}^{d}$, and let (strong) complex frames $\mathcal{H}^{0}=\left\{H_{1}^{0}, \ldots, H_{d}^{0}\right\}$ and $\mathcal{H}^{1}=\left\{H_{1}^{1}, \ldots, H_{d}^{1}\right\}$ be given for $U$, with $\bigcap_{i=1}^{m} H_{i}^{t}=U$ and $\bigcap_{i=1}^{d} H_{i}^{t}=\{0\}$ for $t=0,1$. We include the hyperplanes $H_{m+1}^{t}, \ldots, H_{d}^{t}$ in the frames since we assumed strong complex frames to be essential. We choose to work with the coarsest possible stratifications of $\mathbb{C}^{d}$, namely those induced by the arrangements $\mathcal{H}^{0}$, resp. $\mathcal{H}^{1}$. Let $c_{\mathcal{H}^{0}}, c_{\mathcal{H}^{1}}$ denote the standard generating cocycles on the simplicial models $\Delta\left(P_{\mathcal{H}^{0}} \backslash P_{\operatorname{link}(\{U\})}\right)$, resp. $\Delta\left(P_{\mathcal{H}^{1}} \backslash P_{\operatorname{link}(\{U\})}\right)$. 
Consider linear bases $\left\{h_{1}^{0}, \ldots, h_{d}^{0}\right\}$ and $\left\{h_{1}^{1}, \ldots, h_{d}^{1}\right\}$ of $\mathbb{C}^{d}$, given by unit normal vectors $h_{i}^{t}$ on $H_{i}^{t}$ for $i=1, \ldots, d, t=0,1$, and define a complex coordinate transformation $T$ on $\mathbb{C}^{d}$ by $T\left(h_{i}^{1}\right)=h_{i}^{0}$ for $i=1, \ldots, d$. This transformation respects the stratifications induced by $\mathcal{H}^{1}$, resp. $\mathcal{H}^{0}$, and restricts to a cellular map between the respective $\mathrm{CW}$-decompositions of the unit sphere. In particular, $T^{\sharp}\left(c_{\mathcal{H}^{0}}\right)=c_{\mathcal{H}^{1}}$ follows from the explicit definition of the cocycles. By restriction, $T$ induces a complex coordinate transformation on the orthogonal space of $U$. Let $\xi \in H^{2 m}\left(U^{\perp}, U^{\perp} \backslash\{0\}\right)$ denote the cohomological dual of the orientation class on $U^{\perp}$ [MS, §9]. A complex coordinate transformation is orientation preserving, hence the map induced by $T$ on $H^{2 m}\left(U^{\perp}, U^{\perp} \backslash\{0\}\right)$ maps $\xi$ to itself. Using naturality of the exact sequence for the pair $\left(U^{\perp}, U^{\perp} \backslash\{0\}\right)$, we conclude that $T$ induces the identity on $H^{2 m-1}\left(U^{\perp} \backslash\{0\}\right) . U^{\perp} \backslash\{0\}$ being a deformation retract of $\mathcal{M}(\{U\})$, another naturality argument implies that $T$ induces the identity on $H^{2 m-1}(\mathcal{M}(\{U\}))$. From this, we conclude that $\left[c_{\mathcal{H}^{0}}\right]=\left[c_{\mathcal{H}^{1}}\right]$. By Proposition 4.5 this identity then translates to corresponding cocycles on any simplicial model for $\mathcal{M}(\{U\})$ that is induced by a hyperplane arrangement which contains both $\mathcal{H}^{0}$ and $\mathcal{H}^{1}$.

For the induction step let $\mathcal{A}=\left\{U_{1}, \ldots, U_{n}\right\}$ be a boolean arrangement of $n$ subspaces and $\widetilde{\mathcal{H}}^{0}, \widetilde{\mathcal{H}}^{1}$ strong complex frames for $\mathcal{A}$ as stated above.

For $|S|<n$, consider the subarrangement $\mathcal{A}_{S}=\left\{U_{i}\right\}_{i \in S}$. Let $c_{\mathcal{A}_{S}, \tilde{\mathcal{H}}_{S}^{t}}, t=0,1$, denote the cocycle defined on $\Delta\left(P_{\mathcal{G}} \backslash P_{\operatorname{link}\left(\mathcal{A}_{S}\right)}\right)$ according to 4.1 with respect to the strong complex frames $\widetilde{\mathcal{H}}_{S}^{t}=\bigcup_{i \in S} \widetilde{\mathcal{H}}_{i}^{t}$ for $\mathcal{A}_{S}$. By induction hypothesis $\left[c_{\mathcal{A}_{S}, \widetilde{\mathcal{H}}_{S}^{0}}\right]=$ $\left[c_{\mathcal{A}_{S}, \widetilde{\mathcal{H}}_{S}^{1}}^{1}\right]$, and Remark 4.4 allows us to transfer this identity to the corresponding cocycles on the complement of $\mathcal{A}$ :

$$
\left[c_{\mathcal{A}, \tilde{\mathcal{H}}^{0}}^{S}\right]=i^{*}\left(\left[c_{\mathcal{A}_{S}, \tilde{\mathcal{H}}_{S}^{0}}^{S}\right]\right)=i^{*}\left(\left[c_{\mathcal{A}_{S}, \tilde{\mathcal{H}}_{S}^{1}}\right]\right)=\left[c_{\mathcal{A}, \tilde{\mathcal{H}}^{1}}^{S}\right] .
$$

For $S=[n]$, we relate the cohomology classes represented by $c_{\mathcal{A}, \tilde{\mathcal{H}}^{0}}^{[n]}, c_{\mathcal{A}, \tilde{\mathcal{H}}^{1}}^{[n]}$ to cohomology classes $c_{\mathcal{A}^{\prime \prime}, \widetilde{\mathcal{H}}^{0^{\prime \prime}}}^{[n-1]}, c_{\mathcal{A}^{\prime \prime}, \widetilde{\mathcal{H}}^{1 \prime \prime}}^{[n-1]}$ for the complement of the restriction $\mathcal{A}^{\prime \prime}$ defined with respect to the "restricted" strong complex frames $\widetilde{\mathcal{H}}^{0^{\prime \prime}}, \widetilde{\mathcal{H}}^{1 "}$. By induction hypothesis, $\left[c_{\mathcal{A}^{\prime \prime}, \widetilde{\mathcal{H}}^{0^{\prime \prime}}}^{[n-1]}\right]=\left[c_{\mathcal{A}^{\prime \prime}, \widetilde{\mathcal{H}}^{1 \prime \prime}}^{[n-1]}\right]$ in $H^{*}\left(\mathcal{M}\left(\mathcal{A}^{\prime \prime}\right)\right)$. Lemma 5.4 reveals $\left(\delta^{*}\right)^{-1}$ as a splitting map in the Mayer-Vietoris long exact sequence considered in the proof of Theorem [5.2. We conclude that

$$
\left[c_{\mathcal{A}, \tilde{\mathcal{H}}^{0}}^{[n]}\right]=\left(\delta^{*}\right)^{-1}\left(\left[c_{\mathcal{A}^{\prime \prime}, \tilde{\mathcal{H}}^{0^{\prime \prime}}}^{[n-1]}\right]\right)=\left(\delta^{*}\right)^{-1}\left(\left[c_{\mathcal{A}^{\prime \prime}, \widetilde{\mathcal{H}}^{1 \prime}}^{[n-1]}\right]\right)=\left[c_{\mathcal{A}, \tilde{\mathcal{H}}^{1}}^{[n]}\right] .
$$

For a geometric arrangement $\mathcal{A}$ and an independent set $S$ in $\mathcal{L}(\mathcal{A})$ we defined a cocycle $c^{S}$ on the complement of $\mathcal{A}$ by considering the corresponding cocycle on the complement of a specified maximal boolean subarrangement $\mathcal{A}_{B(S)}$ in $\mathcal{A}$ and restricting it to the complement of $\mathcal{A}$ (cf.5.1). The previous proposition now enables us to show that for $S \in \mathcal{L}(\mathcal{A})$ we can work with any boolean subarrangement in $\mathcal{A}$ that contains $\mathcal{A}_{S}$ and with any strong complex frame - once we adjust Definition 5.1 to the respective setting we reach to a cocycle that represents the same cohomology class as the original cocycle $c^{S}$ :

Corollary 6.2. Let $\mathcal{A}=\left\{U_{1}, \ldots, U_{n}\right\}$ be a geometric arrangement in $\mathbb{C}^{d}$, with a simplicial model for $\mathcal{M}(\mathcal{A})$ as in Definition 5.1. Let $\widetilde{\mathcal{H}}^{0}\left(\mathcal{A}_{T}\right) \subseteq \mathcal{G}$ be an additional strong complex frame for a boolean subarrangement $\mathcal{A}_{T}, T \in \overline{\mathcal{I}}(\mathcal{L}(\mathcal{A}))$. For $S \subseteq T$ 
define

$$
\bar{c}^{S}:=i^{\sharp}\left(c_{\mathcal{A}_{T}}^{S}\right),
$$

where $c_{\mathcal{A}_{T}}^{S}$ denotes the standard cocycle on $\Delta\left(P_{\mathcal{G}} \backslash P_{\operatorname{link}\left(\mathcal{A}_{T}\right)}\right)$ defined with respect to $\widetilde{\mathcal{H}}^{0}\left(\mathcal{A}_{T}\right)$ and $i: \Delta\left(P_{\mathcal{G}} \backslash P_{\operatorname{link}(\mathcal{A})}\right) \longrightarrow \Delta\left(P_{\mathcal{G}} \backslash P_{\operatorname{link}\left(\mathcal{A}_{T}\right)}\right)$ is the natural inclusion. Then, the cohomology classes induced by $\bar{c}^{S}$ and $c^{S}$ coincide:

$$
\left[\bar{c}^{S}\right]=\left[c^{S}\right]
$$

Proof. The inclusion of $\mathcal{M}(\mathcal{A})$ into $\mathcal{M}\left(\mathcal{A}_{S}\right)$ factors through the inclusion into $\mathcal{M}\left(\mathcal{A}_{T}\right)$ and through the inclusion into $\mathcal{M}\left(\mathcal{A}_{B(S)}\right)$ :

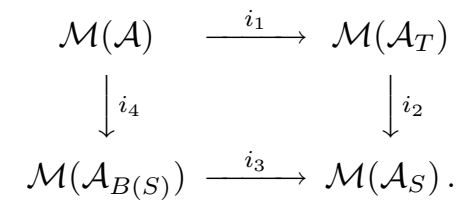

Remark 4.4 combined with the definition of $\bar{c}^{S}$ shows $\left[\bar{c}^{S}\right]=i_{1}^{*} \circ i_{2}^{*}\left(\left[c_{\mathcal{A}_{S}, \tilde{\mathcal{H}}^{0}\left(\mathcal{A}_{S}\right)}\right]\right)$, where the latter cocycle is defined with respect to the subframe $\widetilde{\mathcal{H}}^{0}\left(\mathcal{A}_{S}\right)$ of $\widetilde{\mathcal{H}}^{0}\left(\mathcal{A}_{T}\right)$. Analogously, $\left[c^{S}\right]=i_{4}^{*} \circ i_{3}^{*}\left(\left[c_{\mathcal{A}_{S}, \tilde{\mathcal{H}}\left(\mathcal{A}_{S}\right)}^{S}\right]\right)$, where the latter cocycle is defined with respect to the subframe $\widetilde{\mathcal{H}}\left(\mathcal{A}_{S}\right)$ of $\widetilde{\mathcal{H}}\left(\mathcal{A}_{B(S)}\right)$. Our previous proposition applied to $c_{\mathcal{A}_{S}}^{S}$ and commutativity of the diagram above yield

$$
\begin{aligned}
{\left[\bar{c}^{S}\right]=i_{1}^{*} \circ i_{2}^{*}\left(\left[c_{\mathcal{A}_{S}, \tilde{\mathcal{H}}^{0}\left(\mathcal{A}_{S}\right)}^{S}\right]\right) } & =i_{1}^{*} \circ i_{2}^{*}\left(\left[c_{\mathcal{A}_{S}, \tilde{\mathcal{H}}\left(\mathcal{A}_{S}\right)}^{S}\right]\right) \\
& =i_{4}^{*} \circ i_{3}^{*}\left(\left[c_{\mathcal{A}_{S}, \tilde{\mathcal{H}}\left(\mathcal{A}_{S}\right)}^{S}\right]\right)=\left[c^{S}\right] .
\end{aligned}
$$

The following observation is crucial for our analysis: If a subspace arrangement is contained in a proper linear subspace of the ambient space, then its complement can be viewed as a multiple suspension. Using the standard isomorphism between the (co)homology of a space and the (co)homology of its suspension we can picture cohomology classes for complements of certain arrangements as "suspensions" of cohomology classes for complements of arrangements in a smaller ambient space. This viewpoint will be crucial in the proof of a later proposition.

Proposition 6.3. Let $\mathcal{A}$ be a complex subspace arrangement in $\mathbb{C}^{d}$ and assume that the subspaces of $\mathcal{A}$ are contained in a complex subspace $U$ in $\mathbb{C}^{d}$ of positive codimension $m$. Let $\mathcal{G}$ be a hyperplane arrangement that contains a complex frame for $\mathcal{A}$ and hyperplanes $H_{1}, \ldots, H_{m}$ in $\mathbb{C}^{d}$ with $\bigcap_{i=1}^{m} H_{i}=U$. Besides $\Delta\left(P_{\mathcal{G}} \backslash P_{\operatorname{link}(\mathcal{A})}\right), \mathcal{G}$ induces the simplicial model $\Delta\left(P_{\mathcal{G}_{\lceil U}} \backslash P_{\operatorname{link}(\mathcal{A})}\right)$ for the complement of $\mathcal{A}$ in $U$, and $\Delta\left(P_{\mathcal{G}} \backslash P_{\operatorname{link}(\mathcal{A})}\right)$ is homotopy equivalent to a $2 m$-fold suspension of $\Delta\left(P_{\mathcal{G}_{\lceil U}} \backslash P_{\operatorname{link}(\mathcal{A})}\right)$.

Let c be a cocycle on $\Delta\left(P_{\mathcal{G}_{\lceil U}} \backslash P_{\operatorname{link}(\mathcal{A})}\right)$ in degree $d$, defined by $c:=\sum_{\tau \in T} \alpha_{\tau} \tau^{*}$, where $T$ is a set of $(d+1)$-element chains of cells in $\Gamma_{\mathcal{G}_{\lceil U}} \backslash \Gamma_{\operatorname{link}(\mathcal{A})}$, and $\alpha_{\tau} \in \mathbb{Z}$ for $\tau \in T$. The $2 m$-fold iteration of the standard isomorphism between the cohomology of a space and the cohomology of its suspension maps $[c] \in \widetilde{H}^{d}\left(\Delta\left(P_{\mathcal{G}_{\lceil U}} \backslash P_{\operatorname{link}(\mathcal{A})}\right)\right)$ to a cohomology class in $\widetilde{H}^{d+2 m}\left(\Delta\left(P_{\mathcal{G}} \backslash P_{\operatorname{link}(\mathcal{A})}\right)\right)$, which can be represented by

$$
\operatorname{susp}^{2 m} c:=\sum_{\tau \in T}(-1)^{m} \alpha_{\tau} \sum \hat{\tau}^{*},
$$


where the second sum is over all $(d+2 m+1)$-element chains of cells $\hat{\tau}$ in $\Gamma_{\mathcal{G}} \backslash \Gamma_{\operatorname{link}(\mathcal{A})}$ that coincide with $\tau$ in their initial $d+1$ cells and are elementary ascending along $\left(H_{1}, \ldots, H_{m}\right)$ on their final $2 m$ cells.

Proof. We discuss a single suspension of an arrangement complement and its effect on cohomology. Let $\mathcal{A}$ be a complex subspace arrangement contained in a real hyperplane $U$ that is obtained from a complex hyperplane $H=\operatorname{ker} \ell_{H}, \ell_{H} \in\left(\mathbb{C}^{d}\right)^{*}$, by $U:=\left\{z \in \mathbb{C}^{d}: \operatorname{im} \ell_{H}(z)=0\right\}$. Consider a simplicial model for the complement of $\mathcal{A}$ induced by a hyperplane arrangement $\mathcal{G}$ that contains $H$. Let a cocycle $c$ be defined on $\Delta_{0}:=\Delta\left(P_{\mathcal{G}_{\lceil U}} \backslash P_{\operatorname{link}(\mathcal{A})}\right)$ as stated in the theorem. We aim to describe a representative on $\Delta:=\Delta\left(P_{\mathcal{G}} \backslash P_{\operatorname{link}(\mathcal{A})}\right)$ for the image of [c] under the suspension isomorphism in cohomology. We realize this isomorphism in the context of the simplicial models in question:

Consider the subcomplex in $\Gamma_{\mathcal{G}}$ formed by cells $\theta$ with $\theta(H)=i$ - a regular CW-decomposition of the "upper" hemisphere $\left(S^{2 d-1}\right)^{+}$when considering $U$ as the equator. Denote its face lattice by $P_{\mathcal{G}_{\Gamma\left(\mathrm{im} \ell_{H} \geq 0\right)}}$. The order complex

$$
\Delta^{+}:=\Delta\left(P_{\mathcal{G}_{\left\lceil\left(\mathrm{im} \ell_{H} \geq 0\right)\right.}} \backslash P_{\operatorname{link}(\mathcal{A})}\right)
$$

provides a simplicial model, in fact a deformation retract of $\left(S^{2 d-1}\right)^{+} \backslash \operatorname{link}(\mathcal{A})$ (compare [Mu, Lemma 70.1]). Analogously,

$$
\Delta^{-}:=\Delta\left(P_{\mathcal{G}_{\Gamma\left(\mathrm{im} \ell_{H} \leq 0\right)}} \backslash P_{\operatorname{link}(\mathcal{A})}\right)
$$

provides a simplicial model for $\left(S^{2 d-1}\right)^{-} \backslash \operatorname{link}(\mathcal{A})$. Obviously, $\Delta^{+} \cap \Delta^{-}=\Delta_{0}$. Moreover, $\Delta^{+} \cup \Delta^{-}=\Delta$, since the intersection of subposets of $P_{\mathcal{G}} \backslash P_{\operatorname{link}(\mathcal{A})}$ that define the order complexes $\Delta^{+}$and $\Delta^{-}$is a lower order ideal in $P_{\mathcal{G}} \backslash P_{\operatorname{link}(\mathcal{A})}$. The isomorphism between the cohomology groups of $\Delta^{0}$ and its suspension $\Delta$ is realized by the connecting homomorphism in the cohomological Mayer-Vietoris sequence for the union of spaces $\Delta^{+}$and $\Delta^{-}$, both being contractible:

$$
\longrightarrow \widetilde{H}^{d}\left(\Delta^{+}\right) \oplus \widetilde{H}^{d}\left(\Delta^{-}\right) \longrightarrow \widetilde{H}^{d}\left(\Delta_{0}\right) \stackrel{\delta^{*}}{\longrightarrow} \widetilde{H}^{d+1}(\Delta) \longrightarrow \widetilde{H}^{d+1}\left(\Delta^{+}\right) \oplus \widetilde{H}^{d+1}\left(\Delta^{-}\right) \longrightarrow
$$

A representative for $\delta^{*}[c]$ on $\Delta\left(P_{\mathcal{G}} \backslash P_{\operatorname{link}(\mathcal{A})}\right)$ can be obtained by tracing $c$ on cochain level through the diagram of cochain complexes that results into the long exact Mayer-Vietoris sequence. We refer to [Fe, Prop. 1.6.3] for the details. Here, we only note the description of a representing cocycle $\operatorname{susp} c$ for $\delta^{*}[c]$ :

$$
\operatorname{susp} c:=\sum_{\tau \in T}(-1)^{d+1} \alpha_{\tau} \sum \hat{\tau}^{*},
$$

where the second sum is over all $(d+2)$-element chains of cells $\hat{\tau}$ in $\Gamma_{\mathcal{G}} \backslash \Gamma_{\operatorname{link}(\mathcal{A})}$ that coincide with $\tau$ after deletion of their last cell $\hat{\tau}^{(d+1)}$ and for which $\hat{\tau}^{(d+1)}(H)=i$.

The general case of a $2 m$-fold suspension stated in the theorem follows by iteration of the single suspension thus described.

Remark 6.4. With the previous proposition we can derive Proposition 4.6 from the explicit description of a cohomology generator for a hyperplane complement given by Björner and Ziegler [BZ]: In the setting of Proposition 4.6, we can view $U$ as a hyperplane in $U^{\prime}=\bigcap_{i=2}^{m} H_{i}$, and the complement of $U$ in $\mathbb{C}^{d}$ as a $2(m-1)$-fold suspension of the complement of $U$ in $U^{\prime}$. Following [BZ, Sect. 7], a cohomology generator for the hyperplane complement is represented by $c=\sum_{\tau} \tau^{*}$, where the sum is over all 2-element chains $\tau: \tau^{(0)}<\tau^{(1)}$ in $\Gamma_{\mathcal{G}_{\left\lceil U^{\prime}\right.}} \backslash \Gamma_{\operatorname{link}(\{U\})}$ with $\tau^{(0)}\left(H_{1}\right)=+$ 
and $\tau^{(1)}\left(H_{1}\right)=i$. The representative for the $2(m-1)$-fold "suspension" of this cohomology generator provided by the previous proposition coincides up to sign with the standard cocycle $c^{\{U\}}=c^{1}$ defined according to 4.1.

The construction of cocycles $c^{S}$ for boolean arrangements according to Definition 4.1 depends heavily on the linear order of the index set $S$. In fact, the characteristic sequences of hyperplanes for $S$ change under reordering of the hyperplanes in a strong complex frame.

Example 6.5. Consider the boolean arrangement of subspaces in $\mathbb{C}^{6}$ given by

$$
U_{1}=\left\{z_{1}=z_{2}=0\right\}, \quad U_{2}=\left\{z_{3}=z_{4}=z_{5}=0\right\}, \quad U_{3}=\left\{z_{1}=z_{4}=z_{6}=0\right\} .
$$

Choosing $u_{1}:=e_{2}, u_{2}:=e_{3}$, and $u_{3}:=e_{6}$, we obtain the framing arrangement

$$
V_{1}=\left\{z_{1}=0\right\}, \quad V_{2}=\left\{z_{4}=z_{5}=0\right\}, \quad V_{3}=\left\{z_{1}=z_{4}=0\right\} .
$$

The following hyperplanes form a strong complex frame in the given setting:

$$
\begin{array}{llll}
\widetilde{\mathcal{H}}_{1}: & H_{1,0}=\left\{z_{2}=0\right\}, & H_{1,1}=\left\{z_{1}=0\right\}, & \\
\widetilde{\mathcal{H}}_{2}: & H_{2,0}=\left\{z_{3}=0\right\}, & H_{2,1}=\left\{z_{5}=0\right\}, & H_{2,2}=\left\{z_{4}=0\right\}, \\
\widetilde{\mathcal{H}}_{3}: & H_{3,0}=\left\{z_{6}=0\right\}, & H_{3,1}=\left\{z_{4}=0\right\}, & H_{3,2}=\left\{z_{1}=0\right\} .
\end{array}
$$

Characteristic sequences of hyperplanes for $S=\{2,3\}<$ are

$$
\widetilde{\mathcal{F}}_{2}^{\{2,3\}<}=\left(H_{2,0}, H_{2,1}\right), \quad \widetilde{\mathcal{F}}_{3}^{\{2,3\}<}=\left(H_{3,0}, H_{3,1}, H_{3,2}\right),
$$

whereas under reversed order, $S=\{3,2\}_{<}$, the selection results in

$$
\widetilde{\mathcal{F}}_{3}^{\{3,2\}<}=\left(H_{3,0}, H_{3,2}\right), \quad \widetilde{\mathcal{F}}_{2}^{\{3,2\}}=\left(H_{2,0}, H_{2,1}, H_{2,2}\right) .
$$

There is no evident relation between the cocycles $c^{\{2,3\}}<$ and $c^{\{3,2\}}<$. However, the following proposition enables us to control the effect which the reordering of an index set has on the cohomology class represented by the corresponding standard cocycle.

Proposition 6.6. Let $\mathcal{A}=\left\{U_{1}, \ldots, U_{n}\right\}$ be a boolean arrangement in $\mathbb{C}^{d}$. For an index set $S=\left\{i_{1}, \ldots, i_{k}\right\}<\subseteq[n]$, let $c^{S}$ denote the standard cocycle defined in 4.1 and $c^{\sigma(S)}, \sigma \in \mathfrak{S}_{|S|}$, the cocycle defined analogously with respect to the order $i_{\sigma(1)}<\ldots<i_{\sigma(k)}$ on $S$. Then

$$
\left[c^{\sigma(S)}\right]=\operatorname{sgn} \sigma\left[c^{S}\right]
$$

where $\operatorname{sgn} \sigma$ denotes the sign of the permutation $\sigma$.

Proof. It suffices to show that $\left[c^{\tau(S)}\right]=-\left[c^{S}\right]$ for any transposition $\tau=(r, r+1) \in$ $\mathfrak{S}_{|S|}, r=1, \ldots,|S|-1$. We will work with the subarrangement $\mathcal{A}_{S}=\left\{U_{i}\right\}_{i \in S}$ and compare cochains $c_{\mathcal{A}_{S}}^{\tau(S)}$ and $c_{\mathcal{A}_{S}}^{S}$ defined on $\Delta\left(P_{\mathcal{G}} \backslash P_{\operatorname{link}\left(\mathcal{A}_{S}\right)}\right)$. Once we prove our claim for those, the result transfers to the corresponding cohomology classes on the complement of $\mathcal{A}$ using Remark 4.4.

Our proof is by induction on the cardinality of $S$. For the induction start, set $S=\{1,2\}, \tau=(1,2) \in \mathfrak{S}_{2}$, and denote $c^{12}:=c_{\mathcal{A}_{\{1,2\}}}^{\{1,2\}}, c^{21}:=c_{\mathcal{A}_{\{1,2\}}}^{\tau(\{1,2\})}$ the cochains that are to be compared.

Assume that $\operatorname{codim}_{\mathbb{C}} U_{1}+\operatorname{codim}_{\mathbb{C}} U_{2}=\operatorname{codim}_{\mathbb{C}} U_{\{1,2\}}$ in $\mathcal{A}_{\{1,2\}}$. Denote characteristic sequences of hyperplanes for $\{i\}$, chosen from the strong complex frame 
$\widetilde{\mathcal{H}}=\widetilde{\mathcal{H}}_{1} \cup \widetilde{\mathcal{H}}_{2}$ for $\mathcal{A}_{\{1,2\}}$, with $\widetilde{\mathcal{F}}^{i}$ for $i=1,2$. The following identities hold for characteristic sequences of hyperplanes with respect to the indicated index orders:

$$
\left(\widetilde{\mathcal{F}}_{1}^{\{1,2\}<}, \widetilde{\mathcal{F}}_{2}^{\{1,2\}<}\right)=\left(\widetilde{\mathcal{F}}_{1}, \widetilde{\mathcal{F}}_{2}\right) \quad \text { and } \quad\left(\widetilde{\mathcal{F}}_{2}^{\{2,1\}<}, \widetilde{\mathcal{F}}_{1}^{\{2,1\}<}\right)=\left(\widetilde{\mathcal{F}}_{2}, \widetilde{\mathcal{F}}_{1}\right) .
$$

For the sequence indexed with the respective maximal element of the index set, the claim is obvious. For $\widetilde{\mathcal{F}}_{1}^{\{1,2\}<}$ we compare cardinalities: $\left|\widetilde{\mathcal{F}}_{1}^{\{1,2\}}<\right|=\operatorname{codim}_{\mathbb{C}} U_{\{1,2\}}-$ $\operatorname{codim}_{\mathbb{C}} U_{2}=\operatorname{codim}_{\mathbb{C}} U_{1}=\left|\widetilde{\mathcal{F}}_{1}\right|$. Both $\widetilde{\mathcal{F}}_{1}^{\{1,2\}<}$ and $\widetilde{\mathcal{F}}_{1}$ contain $H_{1,0}$ by definition. Moreover, a hyperplane $H_{1, t}$ that is chosen for $\mathcal{F}_{1}^{\{1,2\}<}$ is also chosen for $\mathcal{F}_{1}$ since $H_{1, t} \nsupseteq \bigcap_{u>t} H_{1, u} \cap V_{2}$ implies that $H_{1, t} \nsupseteq \bigcap_{u>t} H_{1, u}$. Hence, $\mathcal{F}_{1}^{\{1,2\}<} \subseteq \mathcal{F}_{1}$ and we conclude that $\widetilde{\mathcal{F}}_{1}^{\{1,2\}<}=\widetilde{\mathcal{F}}_{1}$. For $\widetilde{\mathcal{F}}_{2}^{\{2,1\}<}$ we argue analogously.

On cochain level the following factorizations hold:

$$
c^{12}=c^{1} \smile c^{2} \quad \text { and } \quad c^{21}=c^{2} \smile c^{1} .
$$

We argue for $c^{12}$ in detail: The cochain $c^{1} \smile c^{2}$ evaluates non-trivially on a chain of cells $\theta: \theta^{(0)}<\ldots<\theta^{\left(2 \operatorname{codim} \mathbb{C} U_{\{1,2\}}-2\right)}$ in $\Gamma_{\mathcal{G}} \backslash \Gamma_{\operatorname{link}\left(\mathcal{A}_{\{1,2\}}\right)}$ if and only if the initial cell sequence of length $2 \operatorname{codim}_{\mathbb{C}} U_{1}-1$ is elementary ascending along $\widetilde{\mathcal{F}}_{1}$, and the final cell sequence of length $2 \operatorname{codim}_{\mathbb{C}} U_{2}-1$ is elementary ascending along $\widetilde{\mathcal{F}}_{2}$ (compare [Br, p. 328] for the explicit evaluation of a cup product on cochain level). These conditions overlap in $\theta^{\left(2 \operatorname{codim}_{\mathbb{C}} U_{1}-1\right)}$ and enforce the sign pattern $\theta^{(t)}\left(\widetilde{\mathcal{F}}_{2}\right)=$ $(+, 0, \ldots, 0)$ on the initial cell sequence, i.e., for $t=0, \ldots, 2 \operatorname{codim}_{\mathbb{C}} U_{1}-1$, and the sign pattern $\theta^{(t)}\left(\widetilde{\mathcal{F}}_{1}\right)=(i, i, \ldots, i)$ on the final cell sequence, i.e., for $t=$ $2 \operatorname{codim}_{\mathbb{C}} U_{1}-1, \ldots, 2 \operatorname{codim}_{\mathbb{C}} U_{\{1,2\}}-2$. It follows that $c^{1} \smile c^{2}$ evaluates nontrivially, namely to $\varepsilon_{2}=-1$, only on chains of cells in $\Gamma_{\mathcal{G}} \backslash \Gamma_{\operatorname{link}\left(\mathcal{A}_{\{1,2\}}\right)}$ that are ascending along $\left(\widetilde{\mathcal{F}}_{1}, \widetilde{\mathcal{F}}_{2}\right)$. By our comparison of characteristic sequences given above, this description coincides with the definition of $c^{12}$ as a sum of elementary cochains. The factorization for $c^{21}$ can be deduced analogously. Thus our assertion now is a simple consequence of the anti-commutativity of the cup product:

$$
\left[c^{21}\right]=\left[c^{2}\right] \smile\left[c^{1}\right]=-\left[c^{1}\right] \smile\left[c^{2}\right]=-\left[c^{12}\right] .
$$

Assume that $\operatorname{codim}_{\mathbb{C}} U_{1}+\operatorname{codim}_{\mathbb{C}} U_{2}>\operatorname{codim}_{\mathbb{C}} U_{\{1,2\}}$ in $\mathcal{A}_{\{1,2\}}$. The codimension of the sum of vector spaces $U_{1}+U_{2}, t:=\operatorname{codim}_{\mathbb{C}}\left(U_{1}+U_{2}\right)$, is strictly positive. We will consider $\mathcal{A}_{\{1,2\}}$ as an arrangement in $U_{1}+U_{2}$ and view its complement in ( $\mathbb{C}^{d}$ as a $2 t$-fold suspension of the complement of $\mathcal{A}_{\{1,2\}}$ in $U_{1}+U_{2}$. Applying Propositions 4.5 and 6.1 we are free to work with a strong complex frame $\widetilde{\mathcal{H}}=\widetilde{\mathcal{H}}_{1} \cup \widetilde{\mathcal{H}}_{2}$ for which the last $t$ hyperplanes in the families $\widetilde{\mathcal{H}}_{1}$ and $\widetilde{\mathcal{H}}_{2}$ coincide and intersect in $U_{1}+U_{2}=V_{1}+V_{2}$. The families of hyperplanes $\widetilde{\mathcal{H}}_{1}^{\prime}=\left\{H_{1, j}: j \leq t_{1}-t\right\}$, $\widetilde{\mathcal{H}}_{2}^{\prime}=\left\{H_{2, j}: j \leq t_{2}-t\right\}$ restricted to $U_{1}+U_{2}$ form a strong complex frame for $\mathcal{A}_{\{1,2\}}$ as an arrangement in $U_{1}+U_{2}$. By the explicit description of representatives for "suspended" cohomology classes given in Proposition 6.3 we see that $c^{12}$ coincides on cochain level with the cocycle $c_{\mathcal{A}_{\left\lceil U_{1}+U_{2}\right.}}^{12}$ "suspended" along the hyperplanes $H_{2, t_{2}-t+1}, \ldots, H_{2, t_{2}}$ up to a coefficient $(-1)^{t}$. Analogously, $c^{21}$ coincides up to $(-1)^{t}$ with the cocycle $c_{\mathcal{A}_{\left\lceil U_{1}+U_{2}\right.}}^{21}$ "suspended" along the (identical) sequence of hyperplanes $H_{1, t_{1}-t+1}, \ldots, H_{1, t_{1}}$. The codimensions of $U_{1}$ and $U_{2}$ in $U_{1}+U_{2}$ add up to the codimension of their intersection. We conclude using our previous considerations:

$$
\left[c^{21}\right]=(-1)^{t}\left[\operatorname{susp}^{2 t} c_{\mathcal{A}_{\left\lceil U_{1}+U_{2}\right.}}^{21}\right]=(-1)^{t+1}\left[\operatorname{susp}^{2 t} c_{\mathcal{A}_{\left\lceil U_{1}+U_{2}\right.}}^{12}\right]=-\left[c^{12}\right] .
$$


For the induction step, let $S=\{1, \ldots, k\}$ and $\tau=(r, r+1)$ in $\mathfrak{S}_{k}$ for $r \in$ $\{1, \ldots, k-1\}$. Assume that $\tau \neq(k-1, k)$. The Mayer-Vietoris argument in the proof of Theorem 5.2 applied to the boolean arrangement $\mathcal{A}_{S}$ reveals $\left[c_{\mathcal{A}_{S}}^{S}\right]$ and $\left[c_{\mathcal{A}_{S}}^{\tau(S)}\right]$ as images of $\left[c_{\mathcal{A}_{S}^{\prime \prime}}^{S \backslash\{k\}}\right]$ and $\left[c_{\mathcal{A}_{S}^{\prime \prime}}^{\tau \backslash\{k\})}\right]$ under the splitting map $\left(\delta^{*}\right)^{-1}$. The latter we can compare by induction hypothesis, and we conclude that

$$
\left[c_{\mathcal{A}_{S}}^{\tau(S)}\right]=\left(\delta^{*}\right)^{-1}\left(\left[c_{\mathcal{A}_{S}^{\prime \prime}}^{\tau(S \backslash\{k\})}\right]\right)=-\left(\delta^{*}\right)^{-1}\left(\left[c_{\mathcal{A}_{S}^{\prime \prime}}^{S \backslash\{k\}}\right]\right)=-\left[c_{\mathcal{A}_{S}}^{S}\right] .
$$

For $\tau=(k-1, k)$, define an arrangement of complex subspaces $\mathcal{W}=\left\{W_{1}, \ldots, W_{k}\right\}$ in $\mathbb{C}^{d}$ by

$$
\begin{array}{lll}
W_{j} & =\bigcap \widetilde{\mathcal{F}}_{j}^{S}, \quad \text { for } j=1, \ldots, k-2, \\
W_{k-1} & =U_{k-1}, & \text { and } \\
W_{k} & =U_{k},
\end{array}
$$

where the $\widetilde{\mathcal{F}}_{j}^{S}$ are characteristic sequences of hyperplanes for $\mathcal{A}_{S}$. The arrangement $\mathcal{W}$ is a boolean arrangement of $k$ subspaces and $\widetilde{\mathcal{H}}^{\mathcal{W}}=\widetilde{\mathcal{F}}_{1}^{S} \cup \ldots \cup \widetilde{\mathcal{F}}_{k-2}^{S} \cup$ $\widetilde{\mathcal{H}}_{k-1} \cup \widetilde{\mathcal{H}}_{k}$ is a complex frame for $\mathcal{W}$; the subframe $\widetilde{\mathcal{H}}_{k-1} \cup \widetilde{\mathcal{H}}_{k}$ we take from $\mathcal{A}_{S}$. The latter is a strong complex frame for the subarrangement $\left\{W_{k-1}, W_{k}\right\}$ in $\mathcal{W}$, whereas we cannot assume in general that $\widetilde{\mathcal{H}}^{\mathcal{W}}$ forms a strong complex frame for $\mathcal{W}$.

Define $c_{\mathcal{W}}^{\{j\}}, j=1, \ldots, k$, to be the standard generating cocycle for the subspace $W_{j}$ on $\Delta\left(P_{\mathcal{G}} \backslash P_{\operatorname{link}(\mathcal{W})}\right)$ with respect to the (strong) complex frame $\widetilde{\mathcal{F}}_{j}^{S}$ for $j \neq k-1, k$, resp. $\widetilde{\mathcal{H}}_{j}$ for $j=k-1, k$. Moreover, define also $c_{\mathcal{W}}^{\{k-1, k\}}$ as in 4.1 For $T=\left\{i_{1}, \ldots, i_{r}\right\}<\subseteq S,|T| \geq 2$, define

$$
c_{\mathcal{W}}^{T}:= \begin{cases}c_{\mathcal{W}}^{\left\{i_{1}\right\}} \smile \ldots \smile c_{\mathcal{W}}^{\left\{i_{r}\right\}} & \text { if }\{k-1, k\} \nsubseteq T, \\ c_{\mathcal{W}}^{\left\{i_{1}\right\}} \smile \ldots \smile c_{\mathcal{W}}^{\left\{i_{r-2}\right\}} \smile c_{\mathcal{W}}^{\{k-1, k\}} & \text { if }\{k-1, k\} \subseteq T .\end{cases}
$$

The proposed cochains are actually cocycles: For $c_{\mathcal{W}}^{\{j\}}, j=1, \ldots, k$, and $c_{\mathcal{W}}^{\{k-1, k\}}$ this follows from Proposition 4.2, all other cochains are cup products of cocycles and hence cocycles themselves. A comparison of descriptions in terms of elementary cochains shows that we obtain identical cochains if we use Definition 4.1 with respect to the (non-strong) complex frame $\widetilde{\mathcal{H}}^{\mathcal{W}}$. The concept of strong complex frames was not used any further in our considerations except to ensure that the cochains defined in 4.1 are actually cocycles. Due to the product structure on the first $k-2$ subspaces in $\mathcal{W}$ we ensure this by our definition of cochains $c_{\mathcal{W}}^{T}$ independently from the frame. In particular, we can conclude as before that the cocycles $c_{\mathcal{W}}^{T}, \emptyset \neq T \subseteq S$, represent a linear basis for $\widetilde{H}^{*}(\mathcal{M}(\mathcal{W}))$.

The cohomology class $\left[c_{\mathcal{W}}^{S}\right]$ factorizes by definition. We apply the induction start on $\left[c_{\mathcal{W}}^{\{k-1, k\}}\right]$ and conclude that

$$
\left[c_{\mathcal{W}}^{\tau(S)}\right]=\left[c_{\mathcal{W}}^{S \backslash\{k-1, k\}}\right] \smile\left[c_{\mathcal{W}}^{\tau(\{k-1, k\})}\right]=-\left[c_{\mathcal{W}}^{S \backslash\{k-1, k\}}\right] \smile\left[c_{\mathcal{W}}^{\{k-1, k\}}\right]=-\left[c_{\mathcal{W}}^{S}\right] .
$$

There is a natural inclusion of arrangement complements, $i: \mathcal{M}(\mathcal{W}) \longrightarrow \mathcal{M}\left(\mathcal{A}_{S}\right)$. By comparison on cochain level we see that $i^{\sharp}\left(c_{\mathcal{A}_{S}}^{S}\right)=c_{\mathcal{W}}^{S}$. Both $c_{\mathcal{A}_{S}}^{S}$ and $c_{\mathcal{W}}^{S}$ represent generating cohomology classes of the top dimensional (infinite cyclic) cohomology group of $\mathcal{M}\left(\mathcal{A}_{S}\right)$, resp. $\mathcal{M}(\mathcal{W})$. Hence, $i^{*}$ is an isomorphism in degree $2 \operatorname{codim}_{\mathbb{C}} \bigcap_{i \in S} U_{i}-|S|$, and our result translates from the complement of $\mathcal{W}$ to the complement of $\mathcal{A}_{S}:\left[c_{\mathcal{A}_{S}}^{\tau(S)}\right]=-\left[c_{\mathcal{A}_{S}}^{S}\right]$. 


\section{The COHOMOlogy Algebras of GeOMETRIC ARRANGEMENTS}

In this section we describe multiplication and derive linear relations among cohomology classes on the complement of a geometric arrangement. This results in a presentation of the cohomology algebra in terms of generators and relations, where cohomology classes $\left[c^{S}\right]$, indexed by independent sets $S$ in $\mathcal{L}(\mathcal{A})$ (cf. Definition 5.1), figure as multiplicative generators.

Proposition 7.1. Let $\mathcal{A}=\left\{U_{1}, \ldots, U_{n}\right\}$ be an arrangement of complex subspaces in $\mathbb{C}^{d}$ with geometric intersection lattice $\mathcal{L}=\mathcal{L}(\mathcal{A})$. Then the cup product of cohomology classes $\left[c^{S}\right],\left[c^{T}\right]$, for $S, T \in \mathcal{I}(\mathcal{L})$, satisfies

$$
\left[c^{S}\right] \smile\left[c^{T}\right]=\left\{\begin{array}{cl}
0, & \text { if } \operatorname{codim}_{\mathbb{C}} U_{S \cup T}<\operatorname{codim}_{\mathbb{C}} U_{S}+\operatorname{codim}_{\mathbb{C}} U_{T}, \\
\operatorname{sgn} \sigma\left[c^{S \cup T}\right], & \text { if } \operatorname{codim}_{\mathbb{C}} U_{S \cup T}=\operatorname{codim}_{\mathbb{C}} U_{S}+\operatorname{codim}_{\mathbb{C}} U_{T},
\end{array}\right.
$$

where $\sigma \in \mathfrak{S}_{|S \cup T|}$ is the permutation which orders $S$ followed by $T$ ascendingly.

Proof. For $S, T \in \mathcal{I}(\mathcal{L})$, assume that $\operatorname{codim}_{\mathbb{C}} U_{S}+\operatorname{codim}_{\mathbb{C}} U_{T}>\operatorname{codim}_{\mathbb{C}} U_{S \cup T}$ and $S \cup T$ is independent in $\mathcal{L}$. We work with the boolean subarrangement $\mathcal{A}_{S \cup T}$. Results on the cup product of $\left[c_{\mathcal{A}_{S \cup T}}^{S}\right]$ and $\left[c_{\mathcal{A}_{S \cup T}}^{T}\right]$ in $H^{*}\left(\mathcal{M}\left(\mathcal{A}_{S \cup T}\right)\right)$ transfer to results on corresponding cohomology classes in $H^{*}(\mathcal{M}(\mathcal{A}))$ via the map induced by the inclusion of complements. In the sequel, we suppress the indices of cocycles that indicate the (sub)arrangement on whose complement a cocycle is defined. The degree of $\left[c^{S}\right] \smile\left[c^{T}\right]$ equals

$$
2 \operatorname{codim}_{\mathbb{C}} U_{S}-|S|+2 \operatorname{codim}_{\mathbb{C}} U_{T}-|T|>2 \operatorname{codim}_{\mathbb{C}} U_{S \cup T}-|S \cup T|,
$$

whereas the maximal degree of a non-trivial cohomology class in $H^{*}\left(\mathcal{M}\left(\mathcal{A}_{S \cup T}\right)\right)$ is $2 \operatorname{codim}_{\mathbb{C}} U_{S \cup T}-|S \cup T|$. We conclude that $\left[c^{S}\right] \smile\left[c^{T}\right]=0$ in $H^{*}\left(\mathcal{M}\left(\mathcal{A}_{S \cup T}\right)\right)$, resp. $H^{*}(\mathcal{M}(\mathcal{A}))$.

For $\operatorname{codim}_{\mathbb{C}} U_{S}+\operatorname{codim}_{\mathbb{C}} U_{T}>\operatorname{codim}_{\mathbb{C}} U_{S \cup T}$, but $S \cup T \notin \mathcal{I}(\mathcal{L})$, we argue via a comparison of degrees in the cohomology of the (non-boolean) subarrangement $\mathcal{A}_{S \cup T}$ : There is a proper subset say of $T, T^{\prime} \subset T$, such that $S \cup T^{\prime}$ is maximal independent in $\mathcal{L}\left(\mathcal{A}_{S \cup T}\right)$. For the degree of $\left[c^{S}\right] \smile\left[c^{T}\right]$ we obtain

$$
\begin{aligned}
\operatorname{deg}\left(\left[c^{S}\right] \smile\left[c^{T}\right]\right) & =2 \operatorname{codim}_{\mathbb{C}} U_{S}-|S|+2 \operatorname{codim}_{\mathbb{C}} U_{T}-|T| \\
& \geq 2 \operatorname{codim}_{\mathbb{C}} U_{S}+2 \operatorname{codim}_{\mathbb{C}} U_{T^{\prime}}-|S|-2\left|T^{\prime}\right|+|T| \\
& \geq 2 \operatorname{codim}_{\mathbb{C}} U_{S \cup T}-\operatorname{rank} \mathcal{L}\left(\mathcal{A}_{S \cup T}\right)-\left|T^{\prime}\right|+|T| \\
& >2 \operatorname{codim}_{\mathbb{C}} U_{S \cup T}-\operatorname{rank} \mathcal{L}\left(\mathcal{A}_{S \cup T}\right),
\end{aligned}
$$

where the first inequality follows from $\operatorname{codim}_{\mathbb{C}} U_{T}-\operatorname{codim}_{\mathbb{C}} U_{T^{\prime}} \geq|T|-\left|T^{\prime}\right|$ for independent sets $T^{\prime} \subseteq T$, and the second from $\operatorname{codim}_{\mathbb{C}} U_{S}+\operatorname{codim}_{\mathbb{C}} U_{T^{\prime}} \geq$ $\operatorname{codim}_{\mathbb{C}} U_{S \cup T^{\prime}}=\operatorname{codim}_{\mathbb{C}} U_{S \cup T}$ and $\operatorname{rank} \mathcal{L}\left(\mathcal{A}_{S \cup T}\right)=|S|+\left|T^{\prime}\right|$. However, the maximal degree of a non-zero cohomology class in $H^{*}\left(\mathcal{M}\left(\mathcal{A}_{S \cup T}\right)\right)$ is $2 \operatorname{codim}_{\mathbb{C}} U_{S \cup T}-$ $\operatorname{rank} \mathcal{L}\left(\mathcal{A}_{S \cup T}\right)$ and we again conclude that $\left[c^{S}\right] \smile\left[c^{T}\right]=0$.

We have left to verify the non-trivial multiplication in case $\operatorname{codim}_{\mathbb{C}} U_{S}+$ $\operatorname{codim}_{\mathbb{C}} U_{T}=\operatorname{codim}_{\mathbb{C}} U_{S \cup T}$. The index sets $S, T \in \mathcal{I}(\mathcal{L})$ are disjoint; otherwise

$\operatorname{codim}_{\mathbb{C}} U_{S \cup T} \leq \operatorname{codim}_{\mathbb{C}} U_{S}+\operatorname{codim}_{\mathbb{C}} U_{T \backslash(S \cap T)}<\operatorname{codim}_{\mathbb{C}} U_{S}+\operatorname{codim}_{\mathbb{C}} U_{T}$.

Moreover, $S \cup T$ is independent in $\mathcal{L}$; if $S \cup T$ were dependent, there exists an element $t$ in $S \cup T$, say $t \in T$, such that $U_{S \cup T}=U_{(S \cup T) \backslash\{t\}}$, and

$$
\operatorname{codim}_{\mathbb{C}} U_{S \cup T} \leq \operatorname{codim}_{\mathbb{C}} U_{S}+\operatorname{codim}_{\mathbb{C}} U_{T \backslash\{t\}}<\operatorname{codim}_{\mathbb{C}} U_{S}+\operatorname{codim}_{\mathbb{C}} U_{T} .
$$


Since we thus restrict our attention to a boolean subarrangement, $\mathcal{A}_{S \cup T}$, we can refer to Corollary 6.2 and assume that all cocycles figuring in the following discussion are defined with respect to a fixed strong complex frame $\widetilde{\mathcal{H}}$ for $\mathcal{A}_{S \cup T}$.

For $S=\left\{i_{1}, \ldots, i_{k}\right\}_{<}$and $T=\left\{j_{1}, \ldots, j_{l}\right\}_{<}$as above, denote by $(S, T)$ the ordered index set $\left\{i_{1}, \ldots, i_{k}, j_{1}, \ldots, j_{l}\right\}_{<}$. Let $c^{(S, T)}$ denote the cocycle defined on $\Delta\left(P_{\mathcal{G}} \backslash P_{\operatorname{link}\left(\mathcal{A}_{S \cup T}\right)}\right)$ according to Definition 4.1 with respect to the indicated linear order on $S \cup T$. Once we show that

$$
\left[c^{S}\right] \smile\left[c^{T}\right]=\left[c^{(S, T)}\right],
$$

our claim follows by applying Proposition 6.6$]$ to $\left[c^{(S, T)}\right]$.

The cocycle $c^{S} \smile c^{T}$ evaluates non-trivially on a chain of cells $\theta$ in $\Gamma_{\mathcal{G}} \backslash \Gamma_{\operatorname{link}}\left(\mathcal{A}_{S \cup T}\right)$ if and only if $\theta$ has an initial cell sequence that is ascending along $\left(\widetilde{\mathcal{F}}_{i_{1}}^{S}, \ldots, \widetilde{\mathcal{F}}_{i_{k}}^{S}\right)$ and a final cell sequence that is ascending along $\left(\widetilde{\mathcal{F}}_{j_{1}}^{T}, \ldots, \widetilde{\mathcal{F}}_{j_{l}}^{T}\right)$. These conditions overlap in one cell and enforce such a chain to be ascending along $\left(\widetilde{\mathcal{F}}_{i_{1}}^{S}, \ldots, \widetilde{\mathcal{F}}_{i_{k}}^{S}, \widetilde{\mathcal{F}}_{j_{1}}^{T}, \ldots, \widetilde{\mathcal{F}}_{j_{l}}^{T}\right)$. We claim that

$$
\left(\widetilde{\mathcal{F}}_{i_{1}}^{S}, \ldots, \widetilde{\mathcal{F}}_{i_{k}}^{S}, \widetilde{\mathcal{F}}_{j_{1}}^{T}, \ldots, \widetilde{\mathcal{F}}_{j_{l}}^{T}\right)=\left(\widetilde{\mathcal{F}}_{i_{1}}^{(S, T)}, \ldots, \widetilde{\mathcal{F}}_{i_{k}}^{(S, T)}, \widetilde{\mathcal{F}}_{j_{1}}^{(S, T)}, \ldots, \widetilde{\mathcal{F}}_{j_{l}}^{(S, T)}\right) .
$$

The straightforward verification will be omitted. We refer to the similar, though slightly simpler reasoning in the proof of Proposition6.6 (for details see [Fe, 1.7.1]).

We conclude that $c^{S} \smile c^{T}$ evaluates non-trivially, in fact to $(-1)^{|S||T|} \varepsilon_{|S|} \varepsilon_{|T|}=$ $\varepsilon_{|S \cup T|}$, on chains of cells in $\Gamma_{\mathcal{G}} \backslash \Gamma_{\operatorname{link}\left(\mathcal{A}_{S \cup T}\right)}$ that are ascending along

$$
\left(\widetilde{\mathcal{F}}_{i_{1}}^{(S, T)}, \ldots, \widetilde{\mathcal{F}}_{i_{k}}^{(S, T)}, \widetilde{\mathcal{F}}_{j_{1}}^{(S, T)}, \ldots, \widetilde{\mathcal{F}}_{j_{l}}^{(S, T)}\right),
$$

a description that coincides with the definition of $c^{(S, T)}$ and thus verifies our claim even on cochain level.

Proposition 7.2. Let $\mathcal{A}=\left\{U_{1}, \ldots, U_{n}\right\}$ be an arrangement of complex subspaces in $\mathbb{C}^{d}$ with geometric intersection lattice $\mathcal{L}=\mathcal{L}(\mathcal{A})$. For any independent set $S$ in $\mathcal{L}$ that contains a broken circuit $C \backslash\left\{i_{1}\right\}$, where $C=\left\{i_{1}, \ldots, i_{k}\right\}<$ is a circuit in $\mathcal{L}$, the following linear relation holds among cohomology classes of degree $2 \operatorname{codim}_{\mathbb{C}} U_{S}-|S|$ :

$$
\sum_{r=1}^{k}(-1)^{r} \operatorname{sgn} \sigma_{r}\left[c^{\left(S \cup\left\{i_{1}\right\}\right) \backslash\left\{i_{r}\right\}}\right]=0,
$$

where $\sigma_{r} \in \mathfrak{S}_{|S|}$ is the permutation that orders $C \backslash\left\{i_{r}\right\}$ followed by $S \backslash\left\{i_{2}, \ldots, i_{k}\right\}$ ascendingly.

Proof. We use induction on the number of elements that are contained in $S$ but not in the broken circuit $C \backslash\left\{i_{1}\right\}$. For the induction start we have to prove an analogue of the classical Orlik-Solomon relations in the cohomology of complex hyperplane arrangements:

$$
\sum_{r=1}^{k}(-1)^{r}\left[c^{C \backslash\left\{i_{r}\right\}}\right]=0,
$$

where $C=\left\{i_{1}, \ldots, i_{k}\right\}$ is a circuit in $\mathcal{L}$.

We work with the subarrangement $\mathcal{A}_{C}=\left\{U_{i}\right\}_{i \in C}$, using again that any linear relation in the cohomology of its complement transfers to the cohomology of the complement of $\mathcal{A}$. An arrangement such as $\mathcal{A}_{C}$ is called a circuit arrangement on $k$ elements: the intersections of less than $k-1$ subspaces are pairwise distinct, whereas all intersections of $k-1$ subspaces coincide. The intersection lattice is a 
boolean algebra on $k$ elements truncated in rank $k-1$. Its associated matroid is the uniform matroid $U_{k-1, k}$, the $k$-element circuit, which explains our terminology. The deletion of a circuit arrangement is boolean, whereas the restriction of a circuit arrangement on $k$ subspaces is a circuit arrangement on $k-1$ subspaces. We allow the degenerate case of a circuit arrangement on two (coinciding) subspaces.

To prove the induction start, we verify the linear relation $(*)$ among the topdimensional cohomology classes on the complement of $\mathcal{A}_{C}$ by induction on the cardinality of $C$. For a circuit arrangement on two subspaces, $\mathcal{C}=\left\{U_{1}, U_{2}\right\}$, the relation states that the generating cocycles $c^{1}, c^{2}$, corresponding to the coinciding subspaces $U_{1}, U_{2}$, and defined with respect to different (strong) complex frames induce the same cohomology class - an assertion that we proved as the induction start in the proof of Proposition 6.1.

Let $\mathcal{C}=\left\{U_{1}, \ldots, U_{k}\right\}$ be a circuit arrangement on $k$ subspaces, $k>2$. By induction hypothesis, the following relation holds in the cohomology of the restriction $\mathcal{C}^{\prime \prime}$ :

$$
\sum_{r=1}^{k-1}(-1)^{r}\left[c_{\mathcal{C}^{\prime \prime}}^{[k-1] \backslash\{r\}}\right]=0 .
$$

Recall from Lemma 5.4 that $\delta^{*}\left(\left[c^{S \cup\{k\}}\right]\right)=\left[c_{\mathcal{C}^{\prime \prime}}^{S}\right]$ for $S \in \mathrm{BC}\left(\mathcal{L}^{\prime \prime}\right)$, hence for index sets $\emptyset \neq S \subset[k-1], S \neq\{2, \ldots, k-1\}$, where $\delta^{*}$ is the connecting homomorphism in the Mayer-Vietoris sequence for the union of $\mathcal{M}\left(\mathcal{C}^{\prime}\right)$ and $\mathcal{M}\left(\left\{U_{k}\right\}\right)$. Similar reasoning verifies a corresponding identity for the index set $S=\{2, \ldots, k-1\}$. Using exactness of the Mayer-Vietoris sequence we conclude

$$
\sum_{r=1}^{k-1}(-1)^{r}\left[c^{[k] \backslash\{r\}}\right] \in \operatorname{ker} \delta^{*}=\operatorname{im} i^{*},
$$

where $\operatorname{im} i^{*} \cong H^{*}\left(\mathcal{M}\left(\mathcal{C}^{\prime}\right)\right) \oplus H^{*}\left(\mathcal{M}\left(\left\{U_{k}\right\}\right)\right)$. The only non-trivial cohomology class in im $i^{*}$ that matches the dimension of the linear combination is $i^{*}\left(\left[c_{\mathcal{C}^{\prime}}^{[k-1]}\right]\right)=$ $\left[c^{[k-1]}\right]$, and we conclude

$$
\sum_{r=1}^{k-1}(-1)^{r}\left[c^{[k] \backslash\{r\}}\right]=\alpha\left[c^{[k-1]}\right] \quad \text { for some } \alpha \in \mathbb{Z} .
$$

We alter the order of subspaces in $\mathcal{C}$ by $\tau=(k-1, k) \in \mathfrak{S}_{k}$ and obtain an analogous linear identity among the top-dimensional cohomology classes on $\mathcal{M}(\mathcal{C})$,

$$
\sum_{r=1}^{k-1}(-1)^{r}\left[c_{\tau}^{[k] \backslash\{r\}}\right]=\beta\left[c_{\tau}^{[k-1]}\right] \quad \text { for some } \beta \in \mathbb{Z},
$$

where $c_{\tau}^{S}$ denotes cocycles that are defined with respect to the permuted order of subspaces. Comparison on cochain level shows that $c_{\tau}^{[k] \backslash\{k\}}=c^{[k] \backslash\{k-1\}}$ and $c_{\tau}^{[k] \backslash\{k-1\}}=c^{[k] \backslash\{k\}}$. Restriction from the complement of the boolean subarrangement $\mathcal{C}_{[k] \backslash\{r\}}$ of $\mathcal{C}$ and an application of Proposition [6.6] yields $\left[c_{\tau}^{[k] \backslash\{r\}}\right]=$

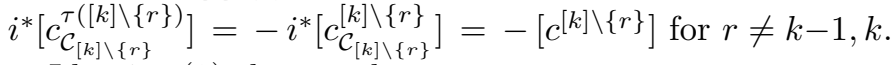

Identity (2) then reads

$$
\sum_{r=1}^{k-2}(-1)^{r+1}\left[c^{[k] \backslash\{r\}}\right]+(-1)^{k-1}\left[c^{[k] \backslash\{k\}}\right]=\beta\left[c^{[k] \backslash\{k-1\}}\right] .
$$


Insertion in (11) yields

$$
\sum_{r=1}^{k-1}(-1)^{r}\left[c^{[k] \backslash\{r\}}\right]=\alpha(-1)^{k-1}\left(\beta\left[c^{[k] \backslash\{k-1\}}\right]+\sum_{r=1}^{k-2}(-1)^{r}\left[c^{[k] \backslash\{r\}}\right]\right) .
$$

The involved cohomology classes form a linear basis for $H^{*}(\mathcal{M}(\mathcal{C}))$ in dimension $2 \operatorname{codim}_{\mathbb{C}} U_{C}-k+1$, as can be seen by a Mayer-Vietoris argument with respect to the linear order $k<1<2<\ldots<k-1$ on the index set of subspaces in $\mathcal{C}$. Comparison of coefficients for $\left[c^{[k] \backslash\{1\}}\right]$ yields $\alpha=(-1)^{k-1}$. Given (1), this finishes the proof of the induction start.

For the induction step let $S$ be an independent set in $\mathcal{L}$ that properly contains the broken circuit $C \backslash\left\{i_{1}\right\}=\left\{i_{2}, \ldots, i_{k}\right\}_{<}, S=\left\{i_{2}, \ldots, i_{k}, j_{1}, \ldots, j_{l}\right\}$. Let us assume for now that $i_{2}<\ldots<i_{k}<j_{1}<\ldots<j_{l}$ in $S$. We work with the subarrangement $\mathcal{B}=\left\{U_{i}\right\}_{i \in S \cup\left\{i_{1}\right\}}$. We claim that $C=\left\{i_{1}, \ldots, i_{k}\right\}$ is the only circuit in $\mathcal{L}(\mathcal{B})$ : Assume $C^{\prime} \neq C$ is another circuit in $\mathcal{L}(\mathcal{B})$. Then $i_{1} \in C^{\prime}$ since otherwise $C^{\prime}$ was contained in $S$ which we assumed to be independent. But if both $C$ and $C^{\prime}$ contain $i_{1}$ there is a circuit $C^{\prime \prime} \subseteq\left(C \cup C^{\prime}\right) \backslash\left\{i_{1}\right\}$ by the circuit elimination axiom for matroids. With $C^{\prime \prime} \subseteq S$ we reach a contradiction. In particular, this reasoning shows that $\left(S \cup\left\{i_{1}\right\}\right) \backslash\left\{i_{r}\right\} \in \mathcal{I}(\mathcal{L})$ for $r=1, \ldots, k$. Since $C$ is a circuit in $\mathcal{L}, U_{C \backslash\left\{i_{1}\right\}}=$ $U_{C \backslash\left\{i_{r}\right\}}$ for $r=2, \ldots, k$, and $U_{\left(S \cup\left\{i_{1}\right\}\right) \backslash\left\{i_{r}\right\}}=U_{C \backslash\left\{i_{r}\right\}} \cap U_{S \backslash\left\{i_{2}, \ldots, i_{k}\right\}}=U_{C \backslash\left\{i_{1}\right\}} \cap$ $U_{S \backslash\left\{i_{2}, \ldots, i_{k}\right\}}=U_{S}$. We conclude that the cocycles indexed by $\left(S \cup\left\{i_{1}\right\}\right) \backslash\left\{i_{r}\right\}$, $r=1, \ldots, k$, are all of degree $2 \operatorname{codim}_{\mathbb{C}} U_{S}-|S|$.

Consider the deletion and the restriction of $\mathcal{B}$. Their intersection lattices have only one circuit as well: For $\mathcal{L}\left(\mathcal{B}^{\prime}\right)$ this is obvious. For $\mathcal{L}\left(\mathcal{B}^{\prime \prime}\right)$ circuits of the contraction are minimal non-empty sets obtained from circuits of the original matroid by removing the contracted elements. In particular, $S \backslash\left\{j_{l}\right\}$ is an independent set in $\mathcal{L}\left(\mathcal{B}^{\prime \prime}\right)$ that contains the (only) broken circuit $C \backslash\left\{i_{1}\right\}$ in $\mathcal{L}\left(\mathcal{B}^{\prime \prime}\right)$ and the number of elements contained in $S \backslash\left\{j_{l}\right\}$ but not in $C \backslash\left\{i_{1}\right\}$ is one less than for $S$ in $\mathcal{L}(\mathcal{B})$.

We can thus apply the induction hypothesis to $\mathcal{B}^{\prime \prime}$ :

$$
\sum_{r=1}^{k}(-1)^{r}\left[c_{\mathcal{B}^{\prime \prime}}^{\left(\left(S \backslash\left\{j_{l}\right\}\right) \cup\left\{i_{1}\right\}\right) \backslash\left\{i_{r}\right\}}\right]=0 .
$$

By exactness of the Mayer-Vietoris sequence on the union of spaces $\mathcal{M}\left(\mathcal{B}^{\prime}\right)$ and $\mathcal{M}\left(\left\{U_{j_{l}}\right\}\right)$ we have

$$
\sum_{r=1}^{k}(-1)^{r}\left[c_{\mathcal{B}}^{\left(S \cup\left\{i_{1}\right\}\right) \backslash\left\{i_{r}\right\}}\right] \in \operatorname{ker} \delta^{*}=\operatorname{im} i^{*},
$$

where $\operatorname{im} i^{*} \cong H^{*}\left(\mathcal{M}\left(\mathcal{B}^{\prime}\right)\right) \oplus H^{*}\left(\mathcal{M}\left(\left\{U_{j_{l}}\right\}\right)\right)$. Top-dimensional classes in these cohomology groups are of dimension $2 \operatorname{codim}_{\mathbb{C}} U_{S \backslash\left\{j_{l}\right\}}-|S|+1$, whereas the degree of the before-mentioned linear combination is strictly larger. We thus conclude that

$$
\sum_{r=1}^{k}(-1)^{r}\left[c_{\mathcal{B}}^{\left(S \cup\left\{i_{1}\right\}\right) \backslash\left\{i_{r}\right\}}\right]=0 .
$$

So far we assumed that $S$ is endowed with a special order, listing first the elements of $C \backslash\left\{i_{1}\right\}$, then the elements of $S \backslash\left\{i_{2}, \ldots, i_{k}\right\}$. Using Proposition [6.6, we can adjust the relation summand by summand to the usual ascending order on $S \cup\left\{i_{1}\right\}$. 
We are now ready to formulate and prove a presentation for the integer cohomology algebras of geometric subspace arrangements in terms of generators and relations.

Theorem 7.3. Let $\mathcal{A}=\left\{U_{1}, \ldots, U_{n}\right\}$ be an arrangement of complex subspaces in $\mathbb{C}^{d}$ with geometric intersection lattice $\mathcal{L}=\mathcal{L}(\mathcal{A})$. The integer cohomology algebra of the complement of $\mathcal{A}$ in $\mathbb{C}^{d}$ is generated by cohomology classes $\left[c^{S}\right], S \in \mathcal{I}(\mathcal{L})$, with representing cocycles as defined in Definition 5.1. It has a presentation as a quotient of the (graded) exterior algebra that is generated by elements $e_{S}$ in dimension $2 \operatorname{codim}_{\mathbb{C}} U_{S}-|S|$ for $S \in \mathcal{I}(\mathcal{L})$,

$$
0 \longrightarrow J \longrightarrow \Lambda^{*}\left(\underset{S \in \mathcal{I}(\mathcal{L})}{\bigoplus} \mathbb{Z}\left[e_{S}\right]\right) \stackrel{\pi}{\longrightarrow} H^{*}(\mathcal{M}(\mathcal{A}) ; \mathbb{Z}) \longrightarrow 0,
$$

where $\pi$ is defined by $\pi\left(e_{S}\right)=\left[c^{S}\right]$. The following elements of the exterior algebra generate the ideal of relations $J$ :

$$
\begin{array}{ll}
e_{S} \wedge e_{T} & \text { for } S, T \in \mathcal{I}(\mathcal{L}) \text { such that } \\
& \quad \operatorname{codim}_{\mathbb{C}} U_{S \cup T}<\operatorname{codim}_{\mathbb{C}} U_{S}+\operatorname{codim}_{\mathbb{C}} U_{T}, \\
e_{S} \wedge e_{T}-\operatorname{sgn} \sigma e_{S \cup T} & \text { for } S, T \in \mathcal{I}(\mathcal{L}) \text { such that } \\
& \quad \operatorname{codim}_{\mathbb{C}} U_{S \cup T}=\operatorname{codim}_{\mathbb{C}} U_{S}+\operatorname{codim}_{\mathbb{C}} U_{T}, \\
& \text { and } \sigma \in \mathfrak{S}_{|S \cup T|} \text { the permutation that orders } \\
& \text { elements of } S \text { followed by elements of } T \text { as- } \\
& \text { cendingly, } \\
& \text { for } S \in \mathcal{I}(\mathcal{L}) \backslash \mathrm{BC}(\mathcal{L}), C=\left\{i_{1}, \ldots, i_{k}\right\}_{<} \text {a } \\
\sum_{r=1}^{k}(-1)^{r} \operatorname{sgn} \sigma_{r} e_{\left(S \cup\left\{i_{1}\right\}\right) \backslash\left\{i_{r}\right\}} & \text { circuit in } \mathcal{L} \text { with } C \backslash\left\{i_{1}\right\} \subseteq S ; \sigma_{r} \in \mathfrak{S}_{|S|} \text { the } \\
& \text { permutation that orders } C \backslash\left\{i_{r}\right\} \text { followed by } \\
& S \backslash\left\{i_{2}, \ldots, i_{k}\right\} \text { ascendingly. }
\end{array}
$$

Proof. There is a linear basis for the cohomology of $\mathcal{M}(\mathcal{A})$ among the multiplicative generators that we propose. By anti-commutativity of the cup product a presentation of the cohomology algebra as a quotient of the exterior algebra on these generators exists. Moreover, we verified the proposed relations among the corresponding cohomology classes, and we are thus left to show that these relations actually generate the ideal $J$.

The relations that describe multiplication among the generators $e_{S}$ obviously reduce the exterior algebra to an algebra that is linearly generated by the elements $e_{S}, S \in \mathcal{I}(\mathcal{L})$. Assume $S \in \mathcal{I}(\mathcal{L})$ contains a broken circuit of $\mathcal{L}$. Due to the additional linear relations, $e_{S}$ can be written as a $\mathbb{Z}$-linear combination of generators with lexicographically smaller index sets. Iterating this process, we write $e_{S}$ as a $\mathbb{Z}$-linear combination of generators that are indexed by elements of the broken circuit complex. Hence, the proposed relations actually reduce the exterior algebra to an algebra which is linearly generated by elements $e_{S}$ with $S \in \mathrm{BC}(\mathcal{L})$. In view of Theorem 5.2, this concludes the proof.

One might suspect that linear relations resembling the classical Orlik-Solomon relations indexed by circuits in $\mathcal{L}$ together with the multiplication rules on the generators $\left[c^{S}\right], S \in \mathcal{I}(\mathcal{L})$, should suffice to generate the ideal of relations $J$. However, the following example shows that the "extended" Orlik-Solomon relations, indexed by independent sets that properly contain a broken circuit in $\mathcal{L}$, are necessary to reduce the exterior algebra to the cohomology algebra of the arrangement. 
Example 7.4. Consider the arrangement $\mathcal{A}$ of four subspaces in $\mathbb{C}^{4}$ given by

$$
\begin{aligned}
& U_{1}=\left\{z_{1}=z_{3}=0\right\} \\
& U_{2}=\left\{z_{2}=z_{3}=0\right\} \\
& U_{3}=\left\{z_{1}+z_{2}=z_{3}=0\right\} \\
& U_{4}=\left\{z_{3}=z_{4}=0\right\}
\end{aligned}
$$

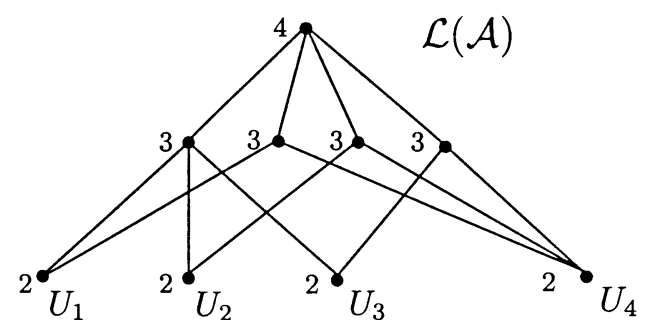

The figure displays the intersection lattice $\mathcal{L}=\mathcal{L}(\mathcal{A})$ without its minimal element, where complex codimensions of the intersections are written next to the corresponding lattice elements.

The following index sets are independent in $\mathcal{L}$ :

$$
\mathcal{I}(\mathcal{L})=\{1,2,3,4,12,13,14,23,24,34,124,134,234\} .
$$

There exists only one circuit $C=\{1,2,3\}$ in $\mathcal{L}$, and the broken circuit complex is

$$
\mathrm{BC}(\mathcal{L})=\{1,2,3,4,12,13,14,24,34,124,134\} .
$$

We list reduced Betti numbers and corresponding generators in the non-trivial dimensions:

\begin{tabular}{l|ccc}
$i$ & 3 & 4 & 5 \\
\hline \hline$\widetilde{\beta}^{i}(\mathcal{M}(\mathcal{A}))$ & 4 & 5 & 2 \\
linear & {$\left[c^{1}\right]\left[c^{2}\right]$} & {$\left[c^{12}\right]\left[c^{13}\right]$} & {$\left[c^{124}\right]\left[c^{134}\right]$} \\
generators & {$\left[c^{3}\right]\left[c^{4}\right]$} & {$\left[c^{14}\right]\left[c^{24}\right]$} & \\
& & {$\left[c^{34}\right]$} &
\end{tabular}

The algebra presentation according to Theorem 7.3 reads:

$$
H^{*}(\mathcal{M}(\mathcal{A}))=\Lambda^{*}\left(\bigoplus_{S \in \mathcal{I}(\mathcal{L})} \mathbb{Z}\left[c^{S}\right]\right) / J
$$

where the ideal of relations $J$ is generated by

$$
\begin{array}{cl}
{\left[c^{S}\right] \smile\left[c^{T}\right]} & \text { for } S, T \in \mathcal{I}(\mathcal{L}), \\
-\left[c^{23}\right]+\left[c^{13}\right]-\left[c^{12}\right], & \text { and } \\
-\left[c^{234}\right]+\left[c^{134}\right]-\left[c^{124}\right] . &
\end{array}
$$

The generating relation which is listed second corresponds to the classical OrlikSolomon relation indexed by the circuit $\{1,2,3\}$ in $\mathcal{L}$. Together with the multiplicative relations it does not suffice to reduce the proposed exterior algebra to an algebra that is isomorphic to the cohomology of the complement of $\mathcal{A}$ : The latter is of rank 2 in degree 5 , whereas there are 3 independent sets in $\mathcal{L}$ that index generators of degree 5 in the exterior algebra. This shows the necessity of the "extended" Orlik-Solomon relation indexed by the independent set $\{2,3,4\}$.

Our investigations cover the case of complex hyperplane arrangements since their intersection lattices are geometric. Cocycles $c^{S}, S \in \mathcal{I}(\mathcal{L})$, as defined in 4.1 respectively 5.1 are in this case products of 1-dimensional cocycles, which are in one-to-one correspondence with the hyperplanes of the arrangement. This makes it 
an easy conclusion to see that our algebra presentation specializes to the classical Orlik-Solomon presentation in the hyperplane case.

Thus we have provided a complete and elementary reproof of the Orlik-Solomon result, avoiding the detour to complex de Rham theory of Björner and Ziegler [BZ] Sect. 7]. Strictly remaining in the context of combinatorial stratifications they had derived the algebra presentation up to the signs in the relations. Their proof holds as well for real 2-arrangements - arrangements of real subspaces of codimension 2 in $\mathbb{R}^{2 d}$ where all intersections have even codimensions (for details on stratifications induced by 2-(pseudo)arrangements see [BZ, Sect. 8]). The ambiguity of signs is a natural limitation for an approach that while dealing with complex hyperplane arrangements does not refer to the complex structure: Though their intersection lattices are geometric, real 2-arrangements are more general than complex hyperplane arrangements - their associated matroids can be non-representable over $\mathbb{C}$ GM, Part III, 5.2], and even the rational cohomology algebra of the complement of a real 2-arrangement is not determined by its combinatorial data [Z, Sect. 2].

Complex structure is essential for our investigations in the induction start of the proof of Proposition 6.1. We show that our description of standard cocycles provides a canonical cohomology generator for a subspace complement. We use the fact that complex coordinate transformations preserve orientation.

We close with an extension of our results to real $(\bmod 2)$-arrangements - arrangements of real subspaces of even codimension in $\mathbb{R}^{2 d}$ where all intersections are as well of even codimension. Combinatorial stratifications induced by real 2-arrangements yield cellular, respectively simplicial models for the complements of real $(\bmod 2)$-arrangements; for details we refer to [Fe, 1.8]. Tracing our arguments in this more general context we can conclude:

Theorem 7.5. The integer cohomology algebra of a real $(\bmod 2)$-arrangement with geometric intersection lattice has a combinatorial presentation in terms of generators and relations as stated in Theorem [7.3; however, the signs in the relations necessarily remain undetermined in the combinatorial context. The analogous presentation for the cohomology algebra with coefficients in $\mathbb{Z}_{2}$ is uniquely determined.

\section{REFERENCES}

[Bj1] A. Björner: Homology and shellability of matroids and geometric lattices; in: Matroid Applications (N. White, ed.); Cambridge University Press, 1992, pp. 226-283. MR 94a:52030

[Bj2] A. Björner: Topological methods; in: Handbook of Combinatorics (R. Graham, M. Grötschel, L. Lovász, eds); North-Holland, Amsterdam, 1995, pp. 1819-1872. MR 96m:52012

[Bj3] A. Björner: Subspace arrangements; in: Proc. First European Congress of Mathematics, Paris 1992 (A. Joseph et al., eds); Birkhäuser, Basel, 1994, pp. 321-370. MR 96h:52012

[Bj4] A. Björner: Nonpure shellability, f-vectors, subspace arrangements and complexity; in: Formal Power Series and Algebraic Combinatorics, DIMACS Workshop 1994 (L.J. Billera et al., eds); Amer. Math. Soc., Providence, R.I., 1996, pp. 25-53. MR 96h:05213

[BZ] A. Björner, G.M. Ziegler: Combinatorial stratification of complex arrangements; J. Amer. Math. Soc. 5 (1992), 105-149. MR 92k:52022

[Br] G.E. Bredon: Topology and Geometry; Graduate Texts in Mathematics 129, SpringerVerlag, 1993. MR 94d:55001

[Bn] E. Brieskorn: Sur les groupes de tresses; in: Séminaire Bourbaki, 1971/1972, no. 401; Lecture Notes in Mathematics, vol. 317, Springer-Verlag, 1973, pp. 21-44. MR 54:10660]

[Bry] T. Brylawski: The broken-circuit complex; Trans. Amer. Math. Soc. 234 (1977), 417-433. MR 80a:05055

[CR] H.H. Crapo, G.-C. Rota: On the Foundations of Combinatorial Geometry: Combinatorial Geometries (preliminary edition); MIT Press, Cambridge, MA, 1970. MR 45:74 
[DP] C. De Concini, C. Procesi: Wonderful models of subspace arrangements; Selecta Math., New Series 1 (1995), 459-494. MR 97k:14013

[Fe] E.M. Feichtner: Cohomology algebras of subspace arrangements and of classical configuration spaces; Doctoral thesis, TU Berlin 1997 (Cuvillier Verlag, Göttingen, 1997).

[GM] M. Goresky, R. MacPherson: Stratified Morse Theory; Ergebnisse der Mathematik und ihrer Grenzgebiete, 3. Folge, Band 14, Springer-Verlag, 1988. MR 90d:57039

[MS] J. Milnor, J. Stasheff: Characteristic Classes; Ann. of Math. Studies, Princeton Univ. Press, 1974. MR 55:13428

[Mu] J.R. Munkres: Elements of Algebraic Topology; Addison-Wesley, 1984. MR 85m:55001

[OS] P. Orlik, L. Solomon: Combinatorics and topology of complements of hyperplanes; Invent. Math. 56 (1980), 167-189. MR 81e:32015

[Ox] J. Oxley: Matroid Theory, Oxford University Press, Oxford, 1992. MR 94d:05033

[Y] S. Yuzvinsky: Small rational model of subspace complement; preprint, March 1998.

[Z] G.M. Ziegler: On the difference between real and complex arrangements; Math. Zeitschrift 212 (1993), 1-11. MR 94f:52017

Department of Mathematics, MA 7-1, TU Berlin, 10623 Berlin, Germany

Current address: Department of Mathematics, ETH Zürich, 8092 Zürich, Switzerland

E-mail address: feichtne@math.ethz.ch

Department of Mathematics, MA 7-1, TU Berlin, 10623 Berlin, Germany

E-mail address: ziegler@math.tu-berlin.de

$U R L$ : www.math.tu-berlin.de/ ziegler 